\title{
S1PR1 regulates the quiescence of lymphatic vessels by inhibiting laminar shear stress-dependent VEGF-C signaling
}

Xin Geng, ${ }^{1}$ Keisuke Yanagida, ${ }^{2}$ Racheal C. Akwii, ${ }^{3}$ Dongwon Choi, ${ }^{4}$ Lijuan Chen, ${ }^{1}$ YenChun Ho, ${ }^{1}$ Boksik Cha, ${ }^{1}$ Md. Riaj Mahamud, ${ }^{1}$ Karen Berman de Ruiz, ${ }^{5}$ Hirotake Ichise, ${ }^{6}$ Hong Chen, ${ }^{2}$ Joshua D. Wythe, ${ }^{5}$ Constantinos M. Mikelis, ${ }^{3}$ Timothy Hla, ${ }^{2}$ and R. Sathish Srinivasan ${ }^{1,7}$

'Cardiovascular Biology Research Program, Oklahoma Medical Research Foundation, Oklahoma City, Oklahoma, USA.

${ }^{2}$ Vascular Biology Program, Boston Children's Hospital, Boston, Massachusetts, USA. ${ }^{3}$ Department of Pharmaceutical Sciences, School of Pharmacy, Texas Tech University Health Sciences Center, Amarillo, Texas, USA. ${ }^{4}$ Keck School of Medicine, University of Southern California, Los Angeles, California, USA. ${ }^{5}$ Cardiovascular Research Institute, Baylor College of Medicine, Houston, Texas, USA. Institute for Animal Research, Faculty of Medicine, University of Ryukyus, Nishihara-cho, Okinawa, Japan. 'Department of Cell Biology, University of Oklahoma Health Sciences Center, Oklahoma City, Oklahoma, USA.

During the growth of lymphatic vessels (lymphangiogenesis), lymphatic endothelial cells (LECs) at the growing front sprout by forming filopodia. Those tip cells are not exposed to circulating lymph, as they are not lumenized. In contrast, LECs that trail the growing front are exposed to shear stress, become quiescent, and remodel into stable vessels. The mechanisms that coordinate the opposed activities of lymphatic sprouting and maturation remain poorly understood. Here, we show that the canonical tip cell marker Delta-like 4 (DLL4) promotes sprouting lymphangiogenesis by enhancing VEGF-C/VEGF receptor 3 (VEGFR3) signaling. However, in lumenized lymphatic vessels, laminar shear stress (LSS) inhibits the expression of DLL4, as well as additional tip cell markers. Paradoxically, LSS also upregulates VEGF-C/VECFR3 signaling in LECs, but sphingosine 1-phosphate receptor 1 (S1PR1) activity antagonizes LSS-mediated VEGF-C signaling to promote lymphatic vascular quiescence. Correspondingly, 51pr1 loss in LECs induced lymphatic vascular hypersprouting and hyperbranching, which could be rescued by reducing Vegfr 3 gene dosage in vivo. In addition, S1PR1 regulates lymphatic vessel maturation by inhibiting RhoA activity to promote membrane localization of the tight junction molecule claudin-5. Our findings suggest a potentially new paradigm in which LSS induces quiescence and promotes the survival of LECs by downregulating DLL4 and enhancing VEGF-C signaling, respectively. S1PR1 dampens LSS/VEGF-C signaling, thereby preventing sprouting from quiescent lymphatic vessels. These results also highlight the distinct roles that S1PR1 and DLL4 play in LECs when compared with their known roles in the blood vasculature.

Conflict of interest: The authors have declared that no conflict of interest exists.

Copyright: (c) 2020, American Society for Clinical Investigation.

Submitted: February 27, 2020

Accepted: June 10, 2020

Published: July 23, 2020.

Reference information: /CI Insight. 2020;5(14):e137652.

https://doi.org/10.1172/jici.

insight.137652.

\section{Introduction}

Several signaling pathways (VEGF-A, Notch, BMP, PDGF-B, angiopoietin-1 and -2, sphingosine 1-phosphate [S1P], and shear stress) are known to regulate angiogenesis in a highly coordinated manner (1). The migratory tip cells and the more mature stalk cells have distinct characteristics (2). One of the important distinctions is that the tip cells are devoid of blood flow, whereas stalk cells actively experience hemodynamic cues and shear stress. Delta-like 4 (DLL4), Adrenomedullin (ADM), Endothelial Cell Specific Marker 1 (ESM1), and Angiopoietin-2 (ANGPT2) are established markers of sprouting, angiogenic tip cells (3). The expression of at least some of these molecules is regulated by VEGF-A, which is expressed maximally in the vicinity of tip cells (4-7). However, to the best of our knowledge, the mechanisms that restrict the expression of these molecules to a few cell layers close to the migrating front are not fully understood.

Growth of lymphatic vessels from preexisting vessels is achieved via lymphangiogenesis, a process that is phenotypically similar to angiogenesis. The mechanisms that control lymphangiogenesis remain incompletely understood (8). VEGF-C binding to, and activation of, its cognate receptor VEGFR3 is the 
most well-studied prolymphangiogenic pathway known and is necessary for the formation, migration, and proliferation of lymphatic endothelial cells (LECs). Indeed, $V e g f c^{-1-}$ mice lack LECs and $V e g f c^{+/-}$mice have severe lymphatic vessel hypoplasia (9). Likewise, mice harboring a dominant negative mutation in VEGFR3 feature hypoplastic lymphatic vessels (10). In contrast, VEGF-C overexpression in mice results in lymphatic vessel overgrowth and dysplasia (11). Consequently, mice overexpressing VEGF-C during a critical developmental time window develop lymphatic vascular defects, such as chylous ascites and chylothorax, and die rapidly after birth. Therefore, a delicate balance of VEGF-C/VEGFR3 signaling is necessary for the proper patterning of the lymphatic vasculature. Specifically, while sprouts must form at the growing front under the influence of VEGF-C, the distal trailing vessels must remain stable and quiescent. Accordingly, negative regulators of VEGF-C/VEGFR3 signaling likely play key roles in coordinating productive lymphatic vascularization.

Shear stress produced by fluid flow is an important regulator of vascular development and physiology (12). Both oscillatory shear stress (OSS) and laminar shear stress (LSS) are necessary for lymphatic vascular development. OSS regulates the expression of molecules such as FOXC2 and GATA2 that are necessary for the maturation of lymphatic vessels and lymphatic valve development (13-16). In contrast, LSS regulates valve maturation (17) and promotes LEC proliferation by inhibiting Notch signaling (18, 19). However, whether any direct crosstalk exists between shear stress and VEGF-C signaling pathways is currently not known.

S1P receptor 1 (S1PR1) is a GPCR that is necessary for preventing hypersprouting of the blood vessel endothelium (20-22). VEGF-A promotes the internalization and degradation of VE-cadherin from tight junctions to promote endothelial sprouting. S1PR1 antagonizes this process by stabilizing VE-cadherin assembly into adherens junctions and by inhibiting VEGF signaling, thus limiting sprouting and enabling blood vessel maturation (20-22). Furthermore, S1PR1 is necessary for normal blood endothelial cell responses to LSS (23). Normally, in response to LSS, blood endothelial cells align in the direction of blood flow and become quiescent (24). However, in the absence of S1PR1, blood endothelial cells fail to align in the direction of flow and do not activate LSS-responsive signals such as phosphorylation of downstream effector molecules like ERK, AKT, and eNOS (23). Based on this knowledge, we hypothesized that S1PR1 also controls lymphatic vascular sprouting during lymphangiogenesis and maturation in response to LSS.

\section{Results}

S1PR1 signaling is active in mature lymphatic vessels. Using our previously reported RNA-Sequencing (RNASeq) data, we determined that S1PR1 is the most strongly expressed S1P receptor in primary cultured human LECs (HLECs) (Supplemental Figure 1A; supplemental material available online with this article; https://doi.org/10.1172/jci.insight.137652DS1) (14, 25). IHC on cryosections from E17.5 WT mouse embryos confirmed that S1PR1 is expressed in LECs in vivo (Supplemental Figure 1B).

S1P binding to S1PR1 stimulates several signaling events such as $\mathrm{G}_{\mathrm{i}}$-dependent Rac GTPase action and $\beta$-arrestin recruitment to the plasma membrane, which leads to receptor internalization. To identify cells with S1PR1 signaling, we employed S1PR1-GFP reporter mice. These mice express (a) S1PR1 C-terminally fused to a tetracycline transactivator (tTA), separated by a Tobacco Etch Virus (TEV) protease recognition site, as well as (b) a $\beta$-arrestin-TEV protease fusion protein. Thus, in the presence of S1P ligand, $\beta$-arrestin-TEV protease is recruited to and cleaves the S1PR1-tTA chimeric receptor; free tTA then translocates to the nucleus to activate expression of a tetracyline response element-driven H2B-EGFP reporter (26). We analyzed cryosections of E16.5 S1PR1-GFP embryos and observed few $\mathrm{GFP}^{+}$blood endothelial cells (Supplemental Figure $1 \mathrm{C}$, arrowheads). Interestingly, most PROX $1^{+} \mathrm{LECs}$ were $\mathrm{GFP}^{+}$(Supplemental Figure 1C, arrows), but some $\mathrm{PROX}^{+}$LECs were clearly GFP ${ }^{-}$(Supplemental Figure $1 \mathrm{C}$, red arrowheads).

To determine whether the $\mathrm{GFP}^{+}$LECs were spatially restricted, we performed whole-mount IHC on the dorsal skin of E16.5 S1PR1-GFP embryos. Lymphatic vessels sprouting from the left and right lymph sacs migrate toward the midline of the dorsal skin in a stereotypic manner (27). These lymphatic vessels featured elevated GFP reporter expression compared with blood vessels (Figure 1A, compare white and yellow arrowheads). Furthermore, GFP was evident in maturing lymphatic vessels (Figure 1A, white arrowheads) and in lymphatic valves (Figure 1A, green arrowheads), but LECs at the migrating front lacked GFP expression (Figure 1A, arrows). As expected, GFP was not expressed in single-transgenic H2B-GFPcontrol littermates (Figure 1A). Thus, S1PR1 signaling is restricted to quiescent LECs in lymphatic vessel 
A S1PR1GFP

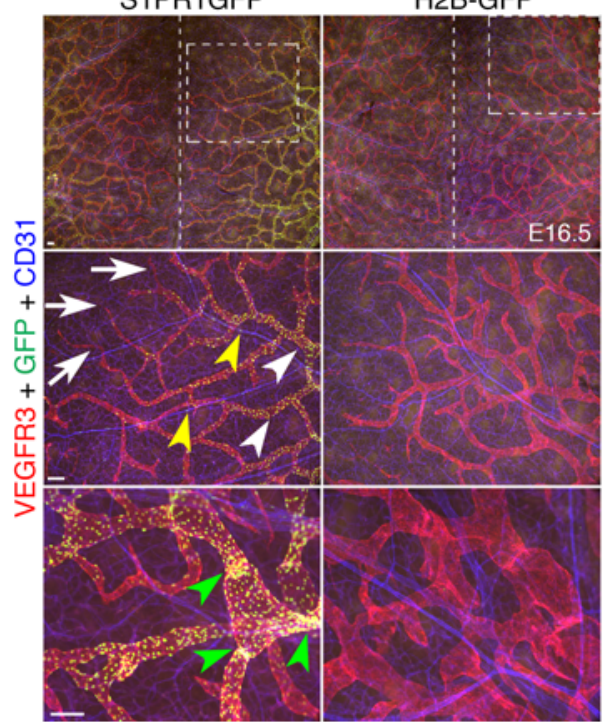

B

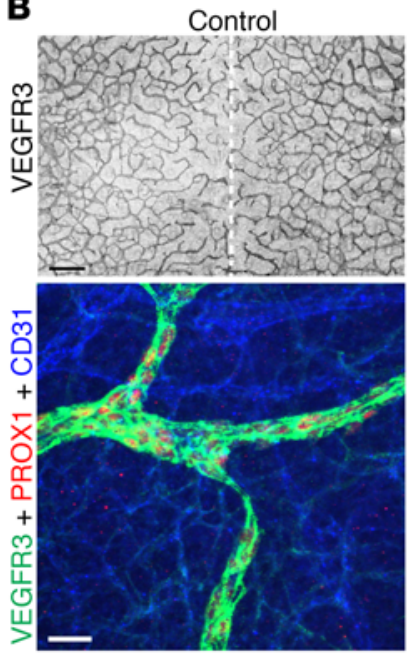

Lyve1-Cre;S1prt-1/F

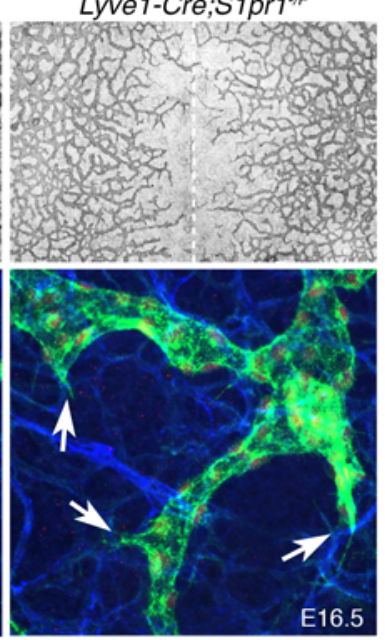

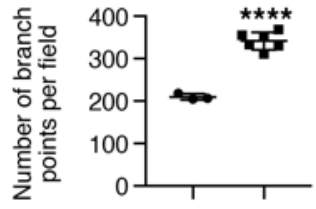

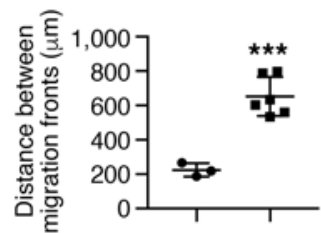

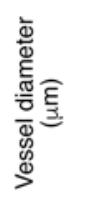

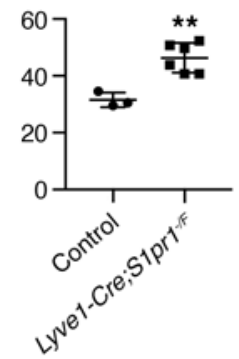

C
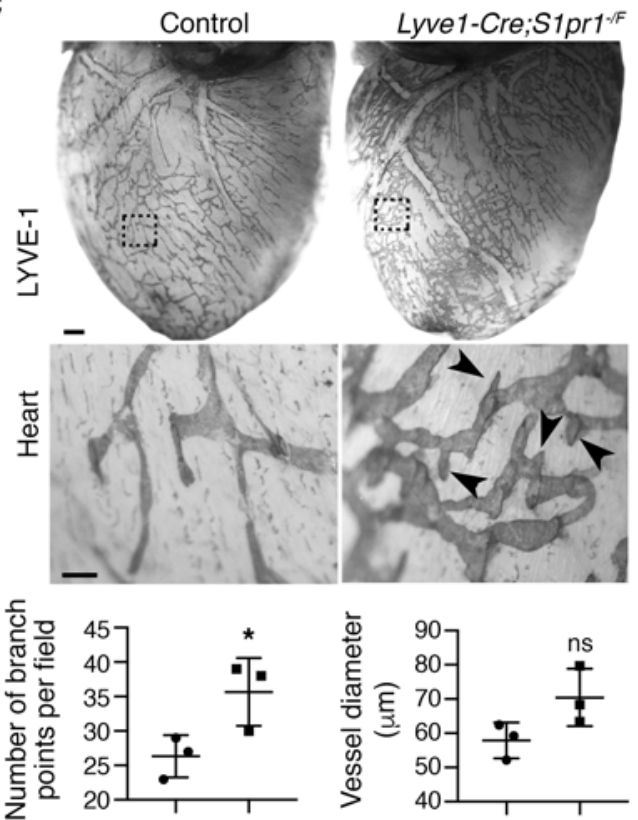

Control
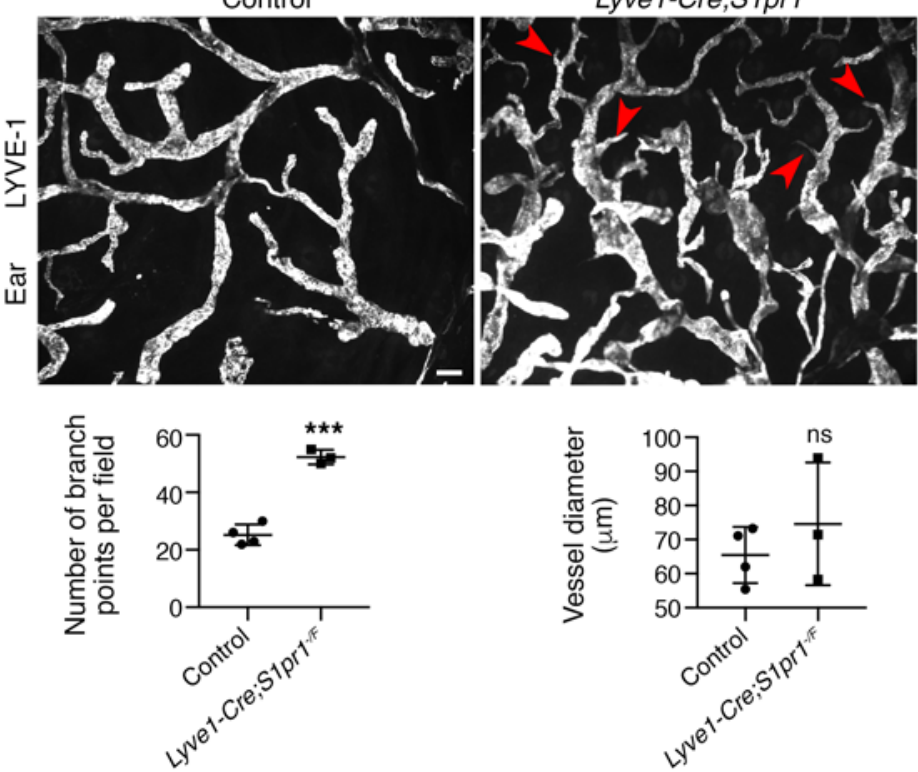

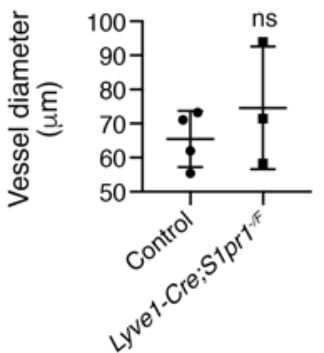

Figure 1. S1PR1 inhibits lymphatic vascular hypersprouting. (A) S1PR1 signaling is active in stable lymphatic vessels and in lymphatic valves. In the dorsal skin, VEGFR $3^{+}$lymphatic vessels migrate from the 2 sides toward the midline (dotted line). H2B-GFP embryos were devoid of GFP expression. Few GFP+ ${ }^{+}$cells were observed in CD31+ blood vessels (yellow arrowheads) or in the migrating front of lymphatic vessels (arrows) in S1PR1-GFP mice. In contrast, LECs that were behind the migrating front (white arrowheads) and lymphatic valves (green arrowheads) were predominantly GFP+. $n=4$ for both S1PR1CFP and H2B-CFP. (B and C) Deletion of S1pr1 from LECs results in lymphatic vascular hypersprouting. (B) The lymphatic vessels of E16.5 embryos lacking S1pr1 were dilated, had excessive number of branches, and did not migrate completely to the midline. Additionally, while the control embryos were devoid of sprouts in between the lymphatic vessel branches, several sprouts were observed in embryos lacking S1pr1 (arrows). $n=3$ for control embryos; $n=6$ for mutant embryos. (C) Heart and ear skin of adult mice lacking S1PR1 had higher lymphatic vessel density and excessive number of sprouts (arrowheads). $n=3$ each per genotype for heart; $n=4$ for control ears and $n=3$ for mutant ears. ${ }^{*} P<0.05,{ }^{* *} P<0.01,{ }^{* * *} P<0.001,{ }^{* * * *} P<0.0001$. Data are presented as mean \pm SEM. Scale bars: (A) $100 \mu \mathrm{M}$; (B, top) $500 \mu \mathrm{M}$; (B, bottom) $50 \mu \mathrm{M}$; (C, heart, top) $500 \mu \mathrm{M}$; (C, heart, bottom) $100 \mu \mathrm{M}$; (C, ear) $100 \mu \mathrm{M}$.

stalks, rather than tip LECs undergoing active lymphangiogenesis. Additionally, a strong S1PR1-GFP signal in LECs when compared with blood vascular endothelial cells suggests that S1PR1 signaling might be biased toward $\beta$-arrestin and G-protein pathways in LECs and blood vascular endothelial cells (28). These results are consistent with a recent report that analyzed LECs and blood vascular endothelial cells from the aorta of adult S1PR1-GFP mice (29). 
S1PR1 prevents lymphatic vessel hypersprouting and hyperbranching. To investigate the function of S1PR1 signaling in the lymphatic endothelium, we used Lyve1-Cre (30) to delete S1pr1 (31) specifically in LECs of the dorsal skin vasculature. We generated Lyve1-Cre;S1pr1-ffl embryos by breeding Lyve1-Cre;S1pr1 ${ }^{+/-}$with S1pr1 ${ }^{f l f l}$ mice. E16.5 Lyve1-Cre;S1pr1 ${ }^{-/ f l}$ embryos had only mild edema, suggesting that the lymphatic drainage is happening in mutant mice (Supplemental Figure 2). However, analysis of the dorsal skin by whole mount IHC revealed that the E16.5 Lyve1-Cre;S1pr1-lfl embryos displayed a higher number of lymphatic vessel branches (Figure 1B). The S1pr1 mutant lymphatic vessels were dilated and did not reach the dorsal midline, unlike control vessels (Figure 1B). The tip cells at the migrating front appeared indistinguishable between control and mutant embryos (Supplemental Figure 3). In contrast, excessive sprouts were seen within the lymphatic plexus of mutant embryos (Figure 1B, arrows).

No obvious recruitment of smooth muscle actin-positive $\left(\mathrm{SMA}^{+}\right)$mural cells were observed on the dermal lymphatic vessels of E16.5 Lyve1-Cre;S1pr1 ${ }^{-f f}$ embryos (data not shown). Fewer numbers of lymphatic valves were seen in the dermal lymphatic vessels of E16.5 Lyve1-Cre;S1pr1-ffl embryos (data not shown). Whether S1PR1 directly regulates lymphatic valve development is currently unknown.

Analysis of the mesenterium of embryonic and early postnatal embryos revealed that lymphatic vessel hyperbranching in Lyve1-Cre;S1pr1-ffl mice (Supplemental Figure 4, A-D). In addition, the lymphatic vessels of the mutant mice were immature, as indicated by increased expression of LYVE1 (Supplemental Figure 4D). Although Lyve1-Cre is specific to the lymphatic vessels of the dermis, it is expressed in the blood vascular endothelial cells of organs such as lungs, liver, and the mesenterium (ref. 32 and our own unpublished observations). Consequently, we also observed hyperbranching of blood vessels in the mesenterium of Lyve1-Cre;S1pr1-ffl mice. Therefore, we cannot exclude the possibility that the hyperbranching of lymphatic and blood vasculatures are functionally related in the mesenterium.

We genotyped over 400 animals at P10 but obtained only 4 Lyve1-Cre;S1pr1-ffl mice, indicating perinatal lethality. The surviving adult animals displayed increased lymphatic vascular sprouts in the heart and the ears (Figure 1C, arrowheads). These results suggest that S1PR1 prevents lymphatic vascular hypersprouting and hyperbranching during embryogenesis and organogenesis.

S1PR 1 regulates the expression of claudin-5 in maturing lymphatic vessels. During angiogenesis, S1PR1 regulates the stability of quiescent blood endothelial cells by promoting the VE-cadherin assembly into adherens junctions $(20,23)$. VE-cadherin is also a mechanosensory molecule that is critical for sensing shear stress (33). Mice lacking Cdh5 (which encodes VE-cadherin) in blood endothelial cells recapitulated the hypersprouting phenotype of mice lacking S1pr1 (20). We did not observe any obvious reduction in VE-cadherin expression within the lymphatic vessels of E17.5 Lyve1-Cre;S1pr1 ${ }^{-/ f l}$ embryos, although VE-cadherin appeared to be mislocalized in some LECs (Figure 2A, red arrowheads). In addition, lymphatic vascular hypersprouting and hyperbranching were not reported in $C d h 5$ lymphatic mutant mice $(34,35)$. Hence, we conclude that VE-cadherin expression is not regulated by S1PR1 in LECs.

S1PR1 maintains the blood-brain barrier by regulating the proper localization of tight junction proteins including claudin-5 (36). Therefore, we tested whether claudin-5 localization could be defective in the lymphatic vessels of Lyve1-Cre;S1pr $1^{-f f}$ embryos. Intriguingly, claudin-5 was expressed in a gradient within the growing lymphatic vessels at E16.5. Claudin-5 expression was lower in the migrating lymphatic vessels compared with the patent vessels of the posterior plexus (Figure 2B, compare red and black arrowheads). This gradient of claudin-5 expression was reminiscent of reporter activity in the S1PR1-GFP embryos (Figure 1A). Therefore, we investigated whether expression of claudin-5 is regulated by S1PR1. Claudin-5 expression remained low in the migrating lymphatic front of Lyve1-Cre;S1pr1-ffl embryos (Figure 2B, red arrowhead). However, claudin-5 was dramatically downregulated in the posterior lymphatic plexus (Figure 2B, black arrowhead). This observation indicated that S1PR1 regulates the expression of claudin-5 in the developing lymphatic vasculature.

Lymphangiography on E17.5 control (Supplemental Figure 5A) and Lyve1-Cre;S1pr1-/fl (Supplemental Figure $5 \mathrm{~B}$ ) embryos did not reveal any obvious leakage of dye in mutant mice, although the excessive branches were clearly demarcated by the dye (Supplemental Figure 5B, arrowheads). Whether the permeability of molecules that are smaller than the dye is increased in the mutant mice remains to be investigated.

In summary, our data suggest that S1PR1 regulates the quiescence and maturation of lymphatic vasculature by preventing hypersprouting and by promoting cell-junction formation, respectively.

DLL4 is a prolymphangiogenic molecule in vitro and in vivo. S1PR1 activity is excluded from the migrating tip cells (Figure 1A), and deletion of S1pr1 from LECs resulted in the dramatic increase in the number of 
A

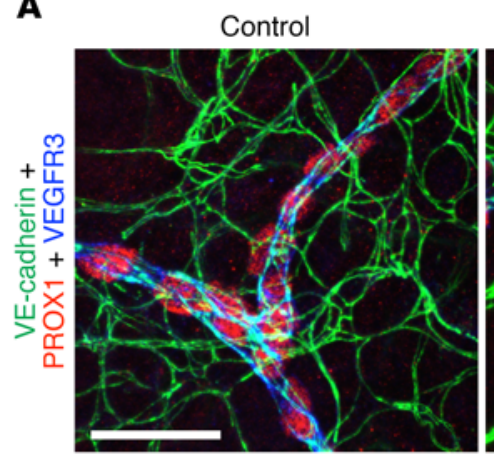

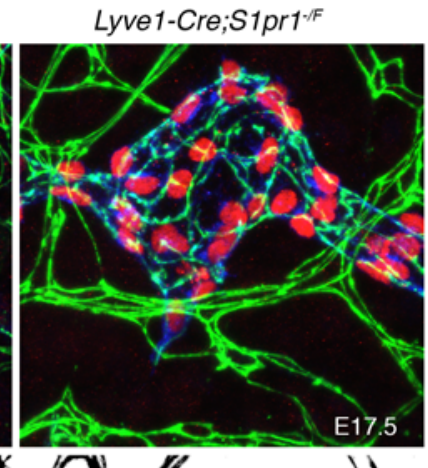

Lyve1-Cre;S1pr1/F

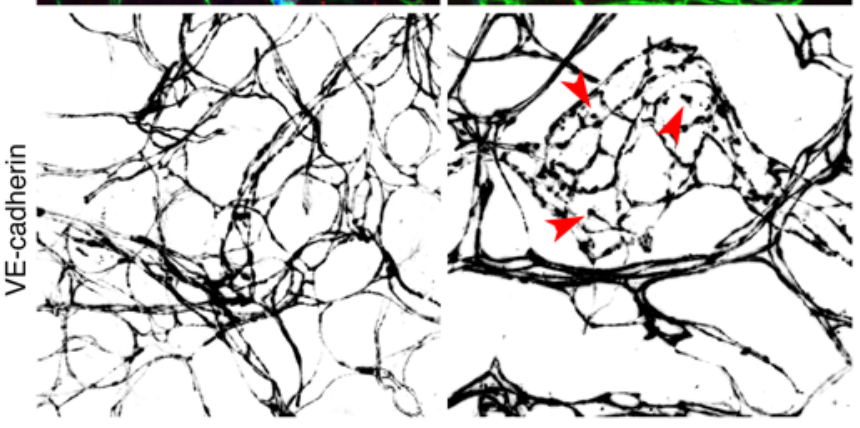

B
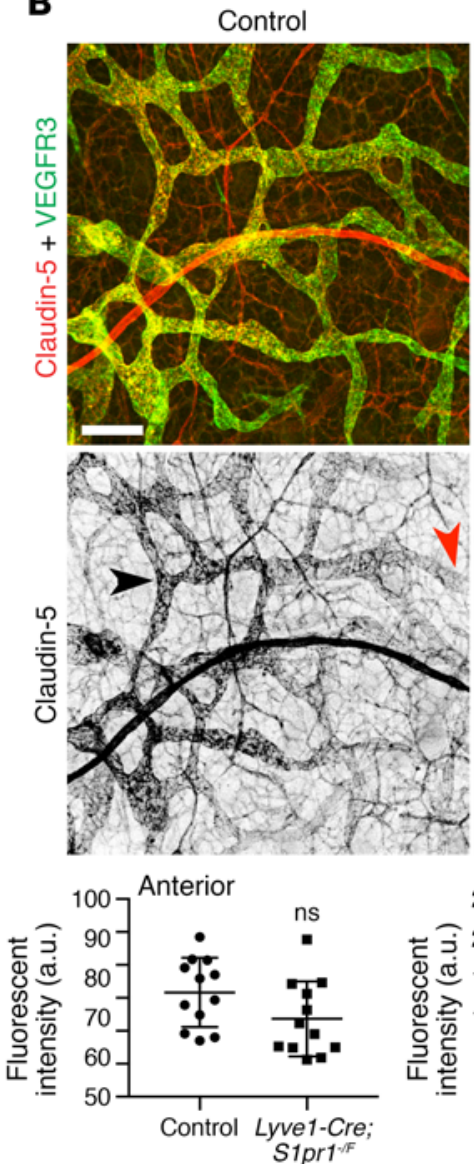

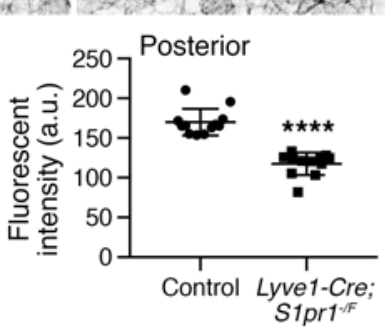

Figure 2. S1PR1 regulates claudin-5 expression in the developing lymphatic vessels. (A) VE-cadherin expression was modestly defective in the lymphatic vessels of embryos lacking S1PR1. VE-cadherin was uniformly expressed with "zipper-like" structure in the lymphatic vessels of E17.5 control embryos. Breaks in VE-cadherin localization were observed (red arrowheads) at restricted locations within the lymphatic vessels of embryos lacking S1PR1. $n=6$ for control embryos; $n$ = 3 for mutant embryos. (B) Claudin- 5 was severely downregulated in embryos lacking S1PR1. In control E16.5 embryos, claudin-5 was expressed in a gradient manner along the developing lymphatic vessels with, weaker expression at the growing tips (red arrowheads) and stronger expression in stable lymphatic vessels behind the migrating front (black arrowheads). In contrast, expression of claudin-5 was uniformly low in embryos lacking S1PR1. $n=6$ for control embryos; $n=10$ for mutant embryos. Fluorescence intensities were measured using $n=3$ samples per genotype. ${ }^{* * * *} P<0.0001$. Data are presented as mean \pm SEM. Scale bars: (A) $50 \mu \mathrm{M}$; (B) $100 \mu \mathrm{M}$.

tip cells (Figure 1B). DLL4 is the prototypic tip cell marker in blood vasculature. Dll4 encodes a Notch ligand that is critical for blood vascular patterning. VEGF-A activation of VEGFR2 in tip cells increases Dll4 expression (5). In turn, DLL4 activates Notch signaling in adjacent stalk cells, which decreases VEGFR2 levels and inhibits excessive sprouting (5, 37, 38). Deletion of just 1 allele of Dll4 results in embryonic death due to arteriovenous malformations and hypersprouting of the blood endothelial cells $(5,37-40)$. DLL4 inhibits VEGF-C signaling in vivo in the zebrafish blood vasculature (41). As in blood vessels, DLL4 is expressed in the tip cells of intestinal lacteals (42). Likewise, DLL4 is expressed in the growing front of dermal lymphatic vessels (Supplemental Figure 6). Both pro- and antilymphangiogenic properties are attributed to DLL4. Overexpression of DLL4-Fc, which inhibits the activity of endogenous DLL4, results in ectopic sprouts (43). In contrast, Dll4 is required for postnatal lymphangiogenesis during wound healing and intestinal lacteal regeneration $(42,44)$. Given that the angiogenic phenotype of $\mathrm{Dll4}^{+/-}$mice is similar to loss of S1PR1, we investigated whether Dll4 is required for normal lymphatic vessel patterning during mouse development.

IHC revealed that the lymphatic vessels in the dorsal skin of E16.5 Dll4 ${ }^{+/-}$embryos were severely hypoplastic (Figure 3A). Additionally, Dll4 ${ }^{+/}$mutant embryo lymphatic vessels were less migratory than control littermates and had significantly fewer branch points (Figure 3A). These results indicate that, in contrast to its role in blood vasculature, DLL4 promotes the growth of lymphatic vessels. VEGF-C and 
A

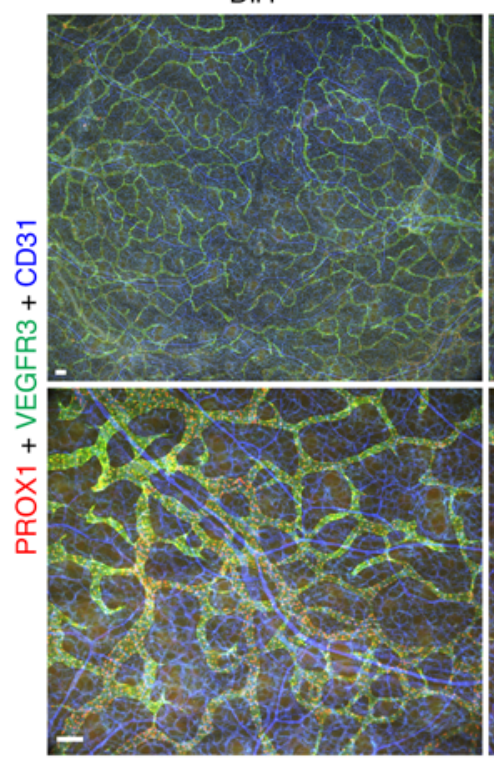

DIII+1-

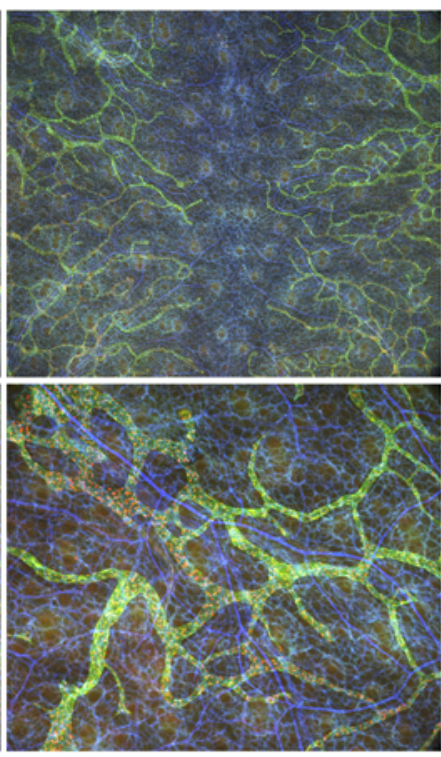

B

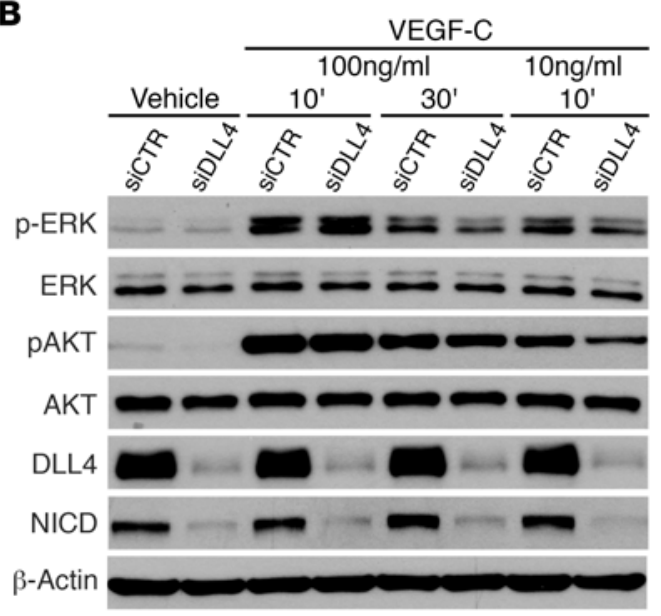

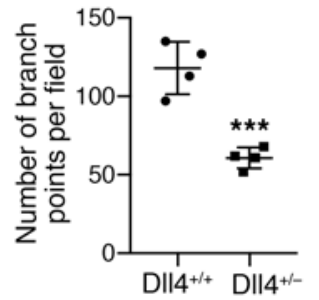

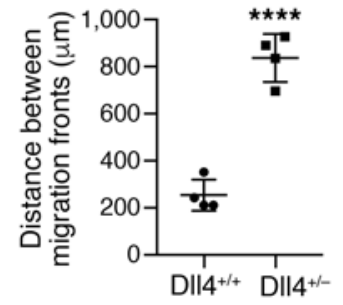

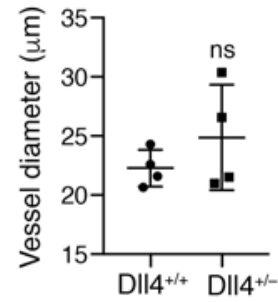

C

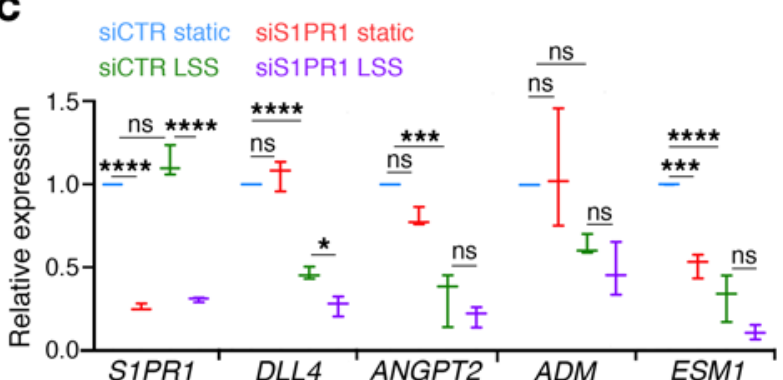

D

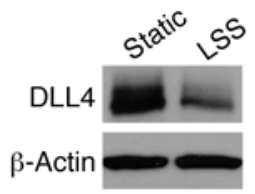

E

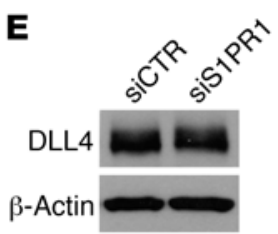

Figure 3. LSS inhibits the expression of prolymphangiogenic tip cell molecules in a S1PR1-independent manner. (A) Lymphatic vessels of DII4+/- mice are hypoplastic. Lymphatic vessels in the dorsal skin of E16.5 DI/4+/- mice had fewer branch points and had migrated lesser distance when compared with their WT littermates. $n=4$ each for WT and DI/4+/- embryos. (B) DLL4 enhances VEGF-C signaling. A signaling pathway activated by VEGF-C was investigated using HLECs that were transfected with either control siRNA or siDLL4. Phosphorylation of ERK and AKT was reduced in HLECs transfected with siDLL4. (C-E) DLL4, ANGPT2, ADM, and ESM1 are inhibited by LSS. HLECs transfected with control siRNA or siS1PR1 were cultured under LSS for 24 hours, and the expression of tip cell markers was quantified by qPCR (C) or by Western blot (D and E). (C) LSS inhibited the expression of all tip cell markers except DLL4 in an S1PR1-independent manner. DLL4 was slightly but significantly more reduced in siS1PR1-transfected HLECs. (D and E) While LSS reduced the expression of DLL4 (D), no obvious differences were was observed between control siRNA and siS1PR1-transfected HLECs that were cultured under LSS (E). $n=3$ for B-E. ${ }^{*} P<0.05,{ }^{* * *} P<0.001,{ }^{* * *} P<0.0001$, 2-tailed unpaired Student's $t$ test (A) or 1-way ANOVA with Bonferroni's correction (C). Data are presented as mean \pm SEM. Scale bars: $100 \mu \mathrm{M}$.

its receptor VEGFR3 are critical, established positive regulators of lymphangiogenesis. Hence, by using in vitro assays, we tested whether DLL4 regulates VEGF-C signaling. Briefly, we transfected HLECs with siDLL4 and then treated them with VEGF-C. As expected, HLECs treated with siDLL4 showed downregulation of DLL4 and Notch1 activation, as assessed by the presence of the cleaved Notch1 intracellular domain (NICD) (Figure 3B). Consistent with the prolymphangiogenic role of DLL4 in vivo, siDLL4 treatment reduced the levels of pERK and pAKT in HLECs treated with VEGF-C (Figure 3B and Supplemental Figure 7, A and B). These results suggest that DLL4 enhances VEGF-C signaling in tip LECs to promote lymphangiogenesis.

LSS inhibits the expression of tip cell-expressed molecules in an S1PR1-independent manner. In addition to DLL4, ANGPT2, ESM1, and ADM are expressed in blood endothelial tip cells (3). Both ANGPT2 and ADM promote lymphatic vascular growth and patterning in vivo $(45,46)$, while ESM1 can enhance HLEC proliferation induced by VEGF-C in vitro (7). Thus, all tip cell-enriched genes are necessary for lymphatic vascular growth. Furthermore, tip cells are not exposed to shear stress, since they lack a functional lumen $(2,22)$. 
We exposed HLECs to 5 dynes $/ \mathrm{cm}^{2}$ LSS as described by Choi et al. $(18,19)$ and investigated whether S1PR1 and LSS could synergize in restricting the expression of tip cell markers. Indeed, LSS potently inhibited the expression of DLL4, ANGPT2, ESM1, and ADM in both control siRNA and siS1PR1-treated HLECs (Figure 3C). Knockdown of S1PR1 slightly reduced the expression of ANGPT2 in HLECs cultured under static conditions (Figure 3C). Expression of ESM1 was also potently downregulated by SiS1PR1 (Figure 3C). However, no obvious differences in expressions of ESM1, ANGPT2, or ADM were observed between control siRNA and siS1PR1-transfected HLECs cultured under LSS (Figure 3C). A slight reduction in DLL4 was observed in siS1PR1-transfected HLECs cultured under LSS (Figure 3C). However, while Western blotting confirmed the downregulation of DLL4 expression by LSS (Figure 3D), we did not observe an obvious difference in DLL4 expression between control siRNA and SiS1PR1-treated HLECs that were cultured under LSS (Figure 3E). These results indicate that LSS inhibits the expression of several tip cell molecules in HLECs in an S1PR1-independent manner.

S1PR1 does not regulate VEGF-C signaling in HLECS cultured under static conditions. Having excluded the role of S1PR1 in regulating tip cell identity, we investigated whether S1PR1 signaling could directly regulate VEGF-C signaling or LSS in HLECs. Briefly, HLECs were serum starved for 6 hours, pretreated for 1 hour with either the S1PR1 agonist SEW2871 or the S1PR1 antagonist W146 (20, 23), and then treated with $100 \mathrm{ng} / \mathrm{mL}$ VEGF-C. We observed no obvious differences in either phosphorylated ERK or AKT between control and SEW2871-treated HLECs, whereas W146 treatment slightly reduced the levels of pERK and pAKT in a statistically nonsignificant manner (Figure 4A and Supplemental Figure 8, A and B). In addition, HLECs treated with control siRNA or SiS1PR1 for 48 hours before stimulation with either 100 $\mathrm{ng} / \mathrm{mL}$ or $10 \mathrm{ng} / \mathrm{mL}$ VEGF-C did not show any obvious differences in pERK or pAKT levels (Figure $4 \mathrm{~B}$, Supplemental Figure 8, C and D, and data not shown). Together, these data suggest that S1PR1 signaling does not inhibit VEGF-C signaling in HLECs under static conditions.

S1PR1 is not necessary for the LSS response in HLECS. LSS promotes the quiescence of blood vascular endothelial cells $(47,48)$. S1PR1 is necessary for HUVEC alignment in response to LSS and for activating LSS-regulated signaling pathways (MEK/ERK, PI3K/AKT, and eNOS) (23). Likewise, LSS elevated pERK and pAKT levels in HLECs within 10 minutes, and this increase returned to baseline levels after 30 minutes (Figure 4C). To determine whether S1PR1 is required for LSS-mediated activation of flow-induced signaling pathways, we exposed HLECs transfected with control siRNA and siS1PR1 to LSS. Phosphorylation of ERK, AKT, p38, and eNOS was indistinguishable between control siRNA and siS1PR1-treated HLECs (Figure 4D and Supplemental Figure 8E). Furthermore, both control and siS1PR1-treated HLECs exposed to 24 hours of LSS had upregulated the shear stress-responsive genes $K L F 2$ and $K L F 4$ (Figure 4E), and were elongated and aligned in the direction of fluid flow (Figure 4F). Altogether, these results demonstrate that S1PR1 is not necessary for canonical LSS responses in HLECs.

LSS enhances VEGF-C signaling, which is antagonized by S1PR1. LSS sensitizes blood endothelial cells to BMP9 and BMP10 signaling (49). To test whether LSS sensitizes HLECs to VEGF-C stimulation, we exposed HLECs to LSS for 24 hours and then treated with $10 \mathrm{ng} / \mathrm{mL}, 5 \mathrm{ng} / \mathrm{mL}$, or $1 \mathrm{ng} / \mathrm{mL}$ of VEGF-C for 10 minutes. We found that LSS enhanced the phosphorylation of ERK and AKT at all tested concentrations of VEGF-C compared with static culture conditions (Figure 5A and Supplemental Figure 9, A and B). Thus, LSS potentiates VEGF-C signaling in HLECs.

To determine whether S1PR1 affects this process, we transfected HLECs with control siRNA or siS1PR1, exposed them to LSS, and then treated them with VEGF-C. We found that phosphorylation of ERK was increased in siS1PR1-treated HLECs compared with control cells (Figure 5B and Supplemental Figure 9C). In contrast, AKT phosphorylation was unchanged (Figure 5B and Supplemental Figure 9D). Total eNOS and phosphorylation of eNOS were also increased in siS1PR1-treated HLECs (Figure 5B, Supplemental Figure 9E, and data not shown). These data suggest that S1PR1 antagonizes specific downstream pathways of LSS-enhanced VEGF-C signaling in HLECs.

Early growth response 1 (EGR1) is an immediate early gene that is activated by VEGF-C in HLECs (4), so we examined whether this process is affected by LSS and S1PR1. EGR1 expression was downregulated by LSS (Figure 5C). Although VEGF-C induced EGR1 expression in both statically cultured and LSS-treated HLECs, the fold induction was higher in HLECs grown under LSS (Figure 5C and Supplemental Figure 10). Importantly, EGR1 was upregulated even further in siS1PR1-treated HLECs compared with control cells that were cultured under LSS. Together, these results further suggest that S1PR1 signaling antagonizes LSS-enhanced VEGF-C signaling. 
A

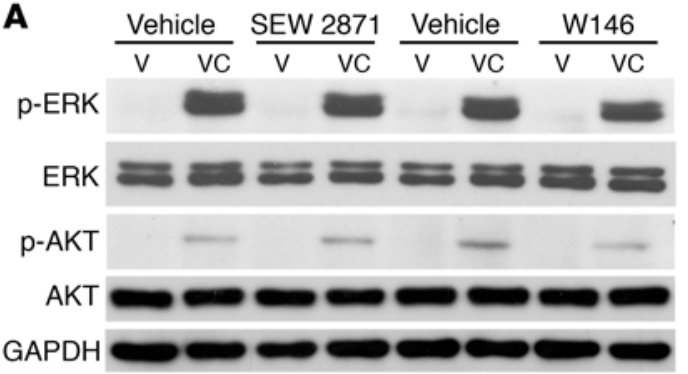

D

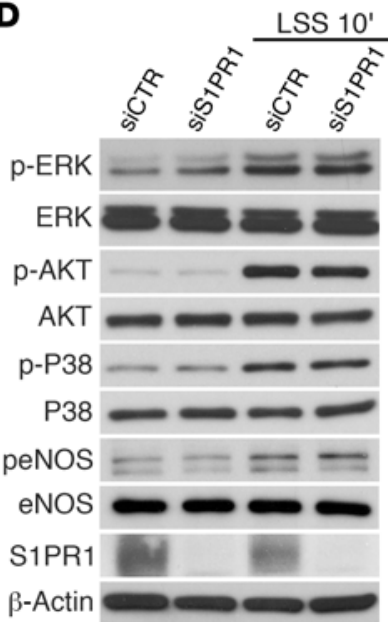

B

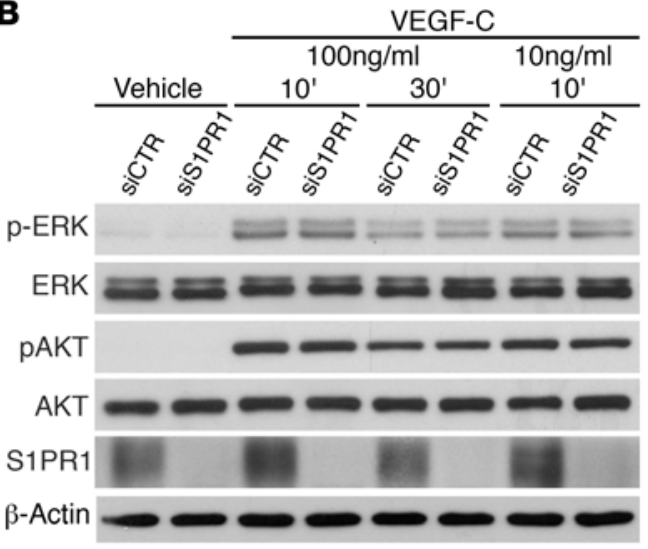

C

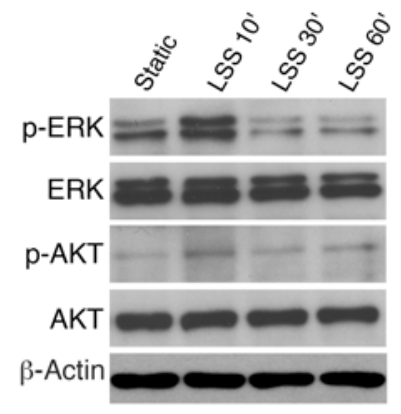

$\mathbf{F}$

E

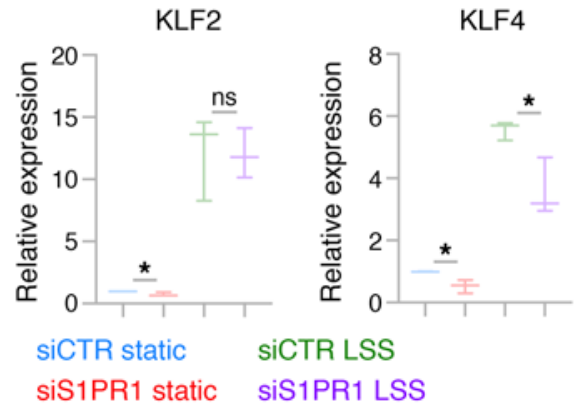

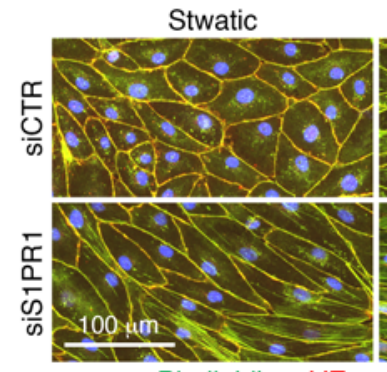

Phalloidin + VE-cadherin + PROX1

Figure 4. S1PR1 does not inhibit VEGF-C signaling in statically cultured HLECs, and it is not necessary for canonical LSS response. (A and B) S1PR1 does not regulate VEGF-C signaling in statically cultured HLECs. (A) HLECs were treated with $100 \mathrm{ng} / \mathrm{mL}$ VEGF-C in the presence or absence of S1PR1 agonist SEW2871 or antagonist W146. SEW2871 did not cause any obvious differences in VEGF-C signaling (phosphorylation of ERK and AKT). W146 slightly reduced the VEGF-C signaling. (B) HLECs were transfected with control siRNA or siS1P1 and then treated with the indicated concentrations of VEGF-C. No obvious differences were observed in VEGF-C signaling between control and siS1PR1-treated HLECs. $n=3$ for A; $n=4$ for B. (C-F) S1PR1 does not regulate canonical LSS-responses in HLECs. (C) Exposure of HLECs to LSS (5 dynes/ $\left.\mathrm{cm}^{2}\right)$ for 10 minutes activated the phosphorylation of ERK and AKT. Prolonged exposure (30-60 minutes) to LSS caused the phosphorylation of ERK and AKT to return to background levels. (D) LSS-induced phosphorylation of ERK, AKT, P38, and eNOS was not affected by siS1PR1. (E) Shear stress responsive genes KLF2 and KLF4 were mildly reduced in siS1PR1-treated HLECs cultured under static conditions. Both KLF2 and KLF4 were upregulated in both control and siS1P1-treated HLECs that were cultured with LSS for 24 hours. A slight reduction in KLF4 expression was observed in siS1PR1-treated HLECs compared with controls. (F) siS1PR1 treatment caused the elongation of statically cultured HLECs. However, both control and siS1PR1-transfected HLECs align normally in the direction of LSS. $n=3$ for $\mathbf{C}, \mathbf{E}$, and $\mathbf{F} ; n=4$ for $\mathbf{D}$. ${ }^{*} P<0.05$. Data are presented as mean \pm SEM. Scale bar: $100 \mu \mathrm{M}$.

Vegfr3 heterozygosity ameliorates hypersprouting caused by the deletion of S1pr1. Given our findings that S1PR1 inhibits VEGF-C/VEGFR3 signaling in vitro, we hypothesized that genetic reduction of VEGF-C/ VEGFR3 signaling components would ameliorate the phenotypes observed in Lyve1-Cre;S1pr1 ${ }^{-f f}$ mice. Indeed, Vegfr3 heterozygosity rescued the increased numbers of sprouts and branches that were observed in lymphatic S1pr1 mutant mice (arrows) (Figure 5D). However, neither the increased vessel diameter nor delayed migration was rescued by Vegfr 3 heterozygosity. Furthermore, claudin-5 expression remained reduced in Lyve1-Cre;S1pr1 ${ }^{-f f} ;$ Vegfr $3^{+/ E G F P}$ embryos (Supplemental Figure 11), suggesting that these SIPRI mutant phenotypes are likely not dependent on VEGF-C/VEGFR3 signaling.

In summary, LSS plays a dual role in regulating lymphangiogenesis. On the one hand, LSS inhibits the expression of prolymphangiogenic molecules such as DLL4, ANGPT2, ESM1, and ADM, and on the other hand LSS, promotes VEGF-C signaling. S1PR1 is not involved in LSS-mediated downregulation of prolymphangiogenic molecules. However, S1PR1 antagonizes LSS-enhanced VEGF-C signaling.

S1PR1 regulates the membrane localization of claudin-5 by inhibiting $R$ hoA. To investigate how S1PR1 regulates claudin-5, we treated cultured HLECs with the S1PR1 inhibitor W146. Thirty minutes of W146 treatment dramatically decreased claudin-5 staining in the periphery of HLECs (Figure 6A). However, Western blotting revealed that the expression of claudin-5 was not downregulated in W146-treated HLECs (data not shown), suggesting that S1PR1 inhibition causes mislocalization of claudin-5. No obvious changes were 
A

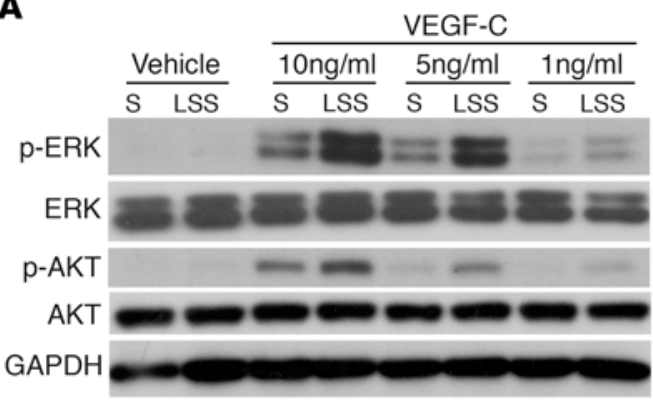

C

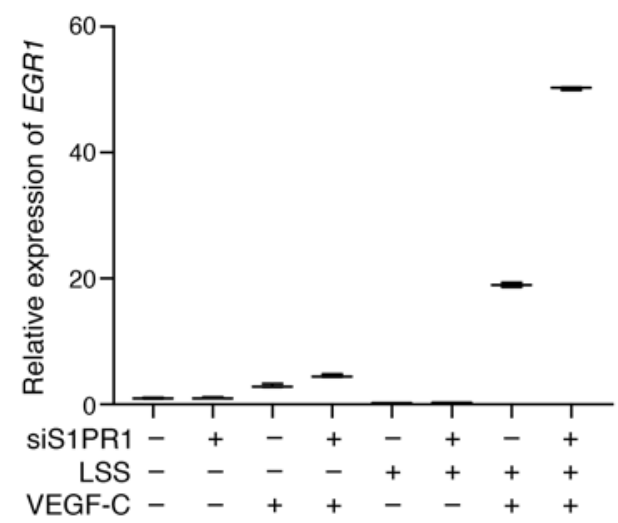

\section{B}

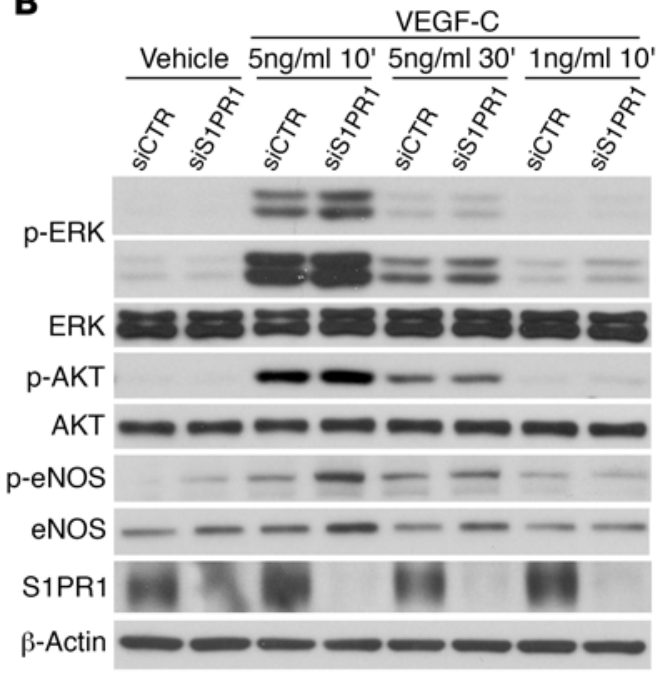

D

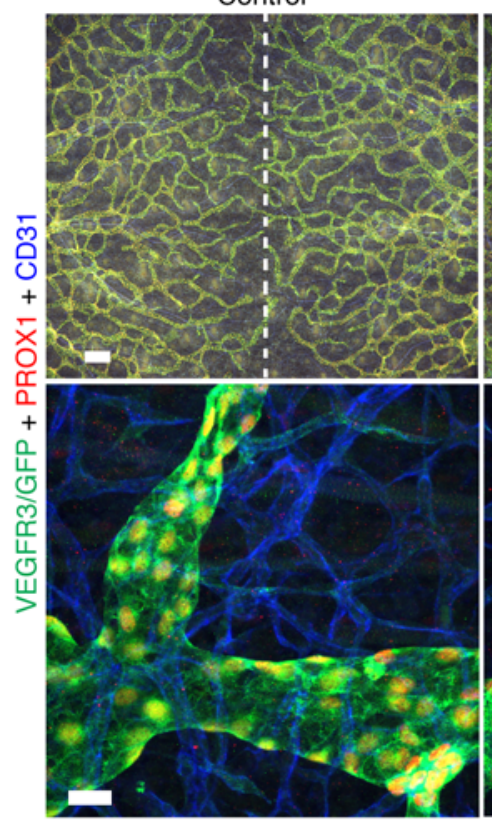

Lyve1-Cre;S1pr1-1F
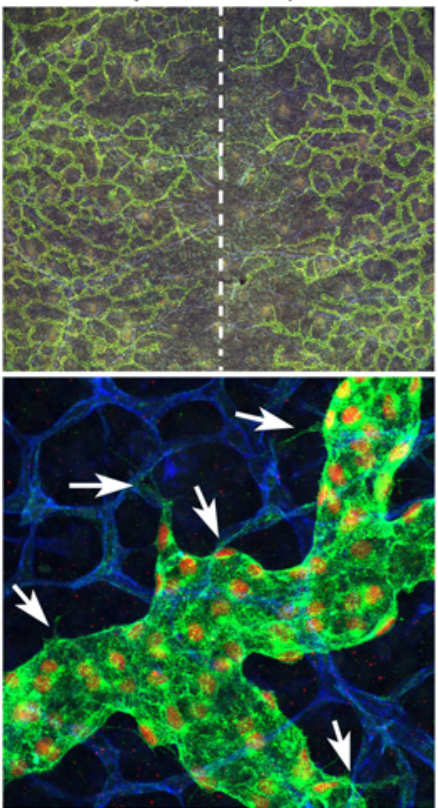

Vegfr3 $^{+ \text {EGFP }} ;$ Lyve1-Cre;S1pr1 ${ }^{-/ F}$

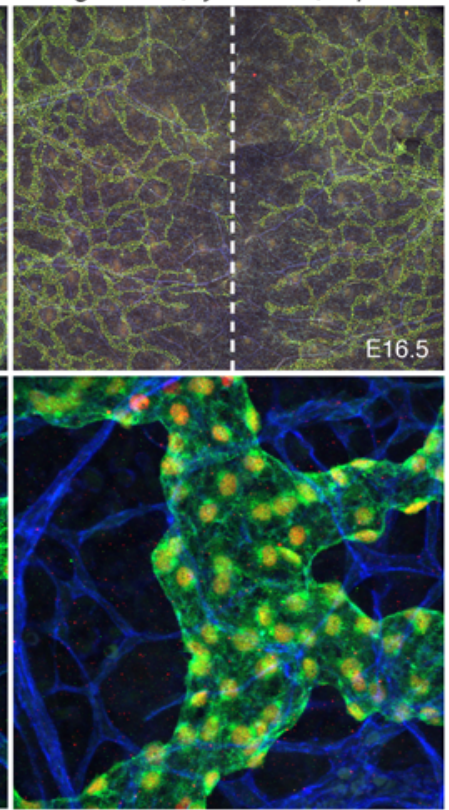

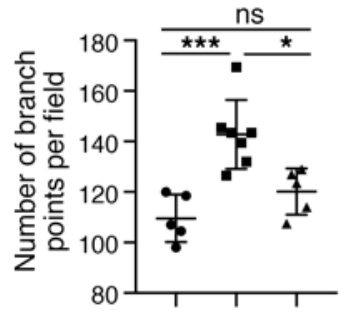
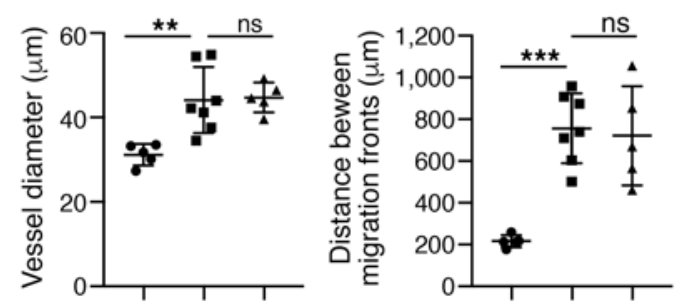

- Control

- Lyve1-Cre;S1pr1-1F

$\Delta$ Vegfr3 ${ }^{+/ E G F P}$. Lyve1-Cre;'S1pr1-1F 
Figure 5. S1PR1 inhibits LSS-enhanced VEGF-C signaling. (A) Exposure to LSS enhances VEGF-C signaling in HLECs. HLECs were cultured with 5 dynes/ $\mathrm{cm}^{2}$ of LSS for 24 hours, following which they were treated with VEGF-C. Phosphorylation of ERK and AKT was enhanced in LSS-exposed HLECs compared with statically cultured cells (S). $n=4$. (B and C) S1PR1 antagonizes LSS enhanced VEGF-C signaling. HLECs were transfected with control siRNA or siS1PR1, exposed to LSS for 24 hours and then treated with VEGF-C for (B) 10 or 30 minutes under static culture condition or for (C) 1 hour in the presence of LSS. (B) Phosphorylation of ERK and eNOS, but not AKT, was enhanced in siS1PR1-transfected HLECs. Total eNOS was also increased in siS1PR1-transfected HLECs. (C) VEGF-C-induced expression of EGR1 was dramatically enhanced by LSS, and it was further enhanced by siS1PR1. $n=4$ for B; C is representative of 3 independent experiments. Results from the other 2 experiments are presented in Supplemental Figure 3. $P$ values were not presented due to large variability in fold activation of EGR1 between independent experiments. (D) Heterozygosity of Vegfr3 rescues the hypersprouting phenotype in embryos lacking S1P1. E16.5 Lyve1-Cre;S1P1-ffl embryos were generated in the Vegfr3-heterozygous background. Increased number of branch points that were observed in Lyve1-Cre;S1P1-ffl embryos was rescued by the loss of 1 allele of Vegfr3. Additionally, the excessive number of sprouts that were seen in Lyve1-

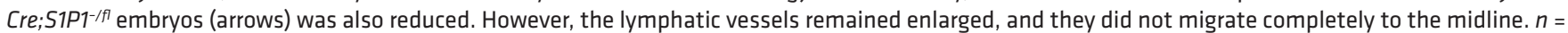
5 for control embryos; $n=7$ for Lyve1-Cre;S1P1-/fl embryos; $n=5$ for Vegfr3 ${ }^{+/ E C F P} ;$ Lyve1-Cre;S1P1 ${ }^{-/ f l}$ embryos. ${ }^{*} P<0.05,{ }^{* *} P<0.01,{ }^{* * *} P<0.001,1$-way ANOVA with Bonferroni's correction (D). Data are presented as mean \pm SEM. Scale bars: (D, top) $100 \mu$ M; (D, bottom) $10 \mu M$.

observed in the expression of other tight junction and adherens junction proteins ZO-1 and VE-cadherin, although they were expressed with a discontinuous (zig-zag) pattern in W146-treated HLECs (Figure 6A and Supplemental Figure 12A). Furthermore, gaps could be seen between cells, suggesting that the cellto-cell interactions were compromised (Supplemental Figure 12A, white arrowheads). We used in vitro cell permeability assays to verify this observation. Briefly, we coated plates with biotin-conjugated gelatin before seeding HLECs. Confluent HLECs were treated with W146 for 30 minutes, fixed, washed, and stained with Alexa 488-conjugated streptavidin. Intercellular gaps were revealed by a green signal. As shown in Supplemental Figure 13, inhibition of S1PR1 by W146 resulted in increased intercellular gaps.

We next investigated how S1PR1 regulates claudin-5 localization. Cell-to-cell interactions and cell shape are regulated by the actin cytoskeleton. Therefore, we analyzed the cytoskeletal architecture of control and W146-treated HLECs by using Alexa 488-conjugated phalloidin staining. Cortical actin (actin around the cell periphery) was enriched in vehicle-treated HLECs (Supplemental Figure 12A). In contrast, treatment with W146 for 30 minutes induced active remodeling of cytoskeleton with the formation of radial actin bundles that are orientated perpendicularly to the cell periphery (Supplemental Figure 12A, red arrowheads). The increased accumulation of the radial actin fibers supports a cell retraction mechanism, which was also supported by the decreased size and compromised barrier integrity of W146-treated HLECs (Supplemental Figure 12A and Supplemental Figure 13). Altogether, these data show that W146 treatment compromises cytoskeletal architecture in cultured LECs.

The Rho family of small GTPases, such as RhoA, regulate cytoskeletal dynamics (50). We found that RhoA activity was increased in HLECs treated with W146 (Figure 6B). We tested whether inhibition of the RhoA/ROCK pathway could restore claudin-5 localization in W146-treated HLECs. Treatment of HLECs with ROCK inhibitor Y-27632 for 6 hours resulted in "oak leaf-shaped" HLECs in which the expression of VE-cadherin and claudin-5 were intact (Figure 6A). Strikingly, Y-27632 pretreatment followed by incubation with W146 for 30 minutes prevented the formation of radial actin bundles, cellular retraction, and claudin-5 downregulation (Figure 6A). These results indicate that S1PR1 regulates the membrane localization of claudin-5 in HLECs by inhibiting RhoA/ROCK activity. Nevertheless, HLEC permeability was increased by Y-27632, as well (Supplemental Figure 13). Furthermore, W146-induced formation of gaps between HLECs was not completely abolished by Y-27632 (Supplemental Figure 12A, white arrowheads and Supplemental Figure 13). These results indicate that both overactivation and inhibition of RhoA activity could compromise HLEC barrier integrity.

Localization of claudin-5 at the periphery of HLECs was restored 24 hours after treatment with W146 (Supplemental Figure 12B). VE-cadherin was also expressed in a uniform manner around the cell membrane, indicating that the cell-to-cell junctions were fully restored. However, treatment with W146 for 24 hours resulted in elongated HLECs with obvious increases in stress fiber (actin fibers that traverse across the cytoplasm) formation (Supplemental Figure 12C). The elongated morphology of HLECs, increased formation of stress fibers, and elevated RhoA activity were also observed in HLECs treated with siSIPRI (Figure 6C and Supplemental Figure 12D). Based on these observations, we conclude that loss of S1PR1 upregulates RhoA activity and transiently disrupts claudin-5 localization and cell-to-cell interactions.

As mentioned above, claudin-5 expression was unaffected in SiS1PR1-treated HLECs before the onset of LSS. Additionally, no obvious differences in claudin- 5 expression were observed between control and SiSIPR1-treated HLECs after exposure to LSS (data not shown). Therefore, we concluded that claudin-5 is dispensable for the crosstalk between LSS and VEGF-C signaling. 
A
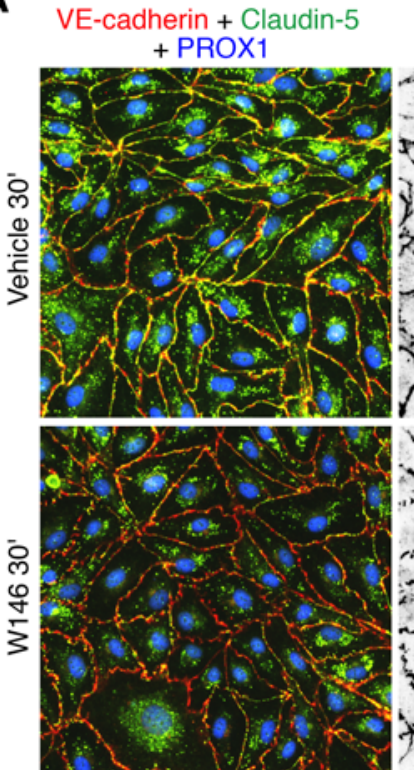

VE-cadherin
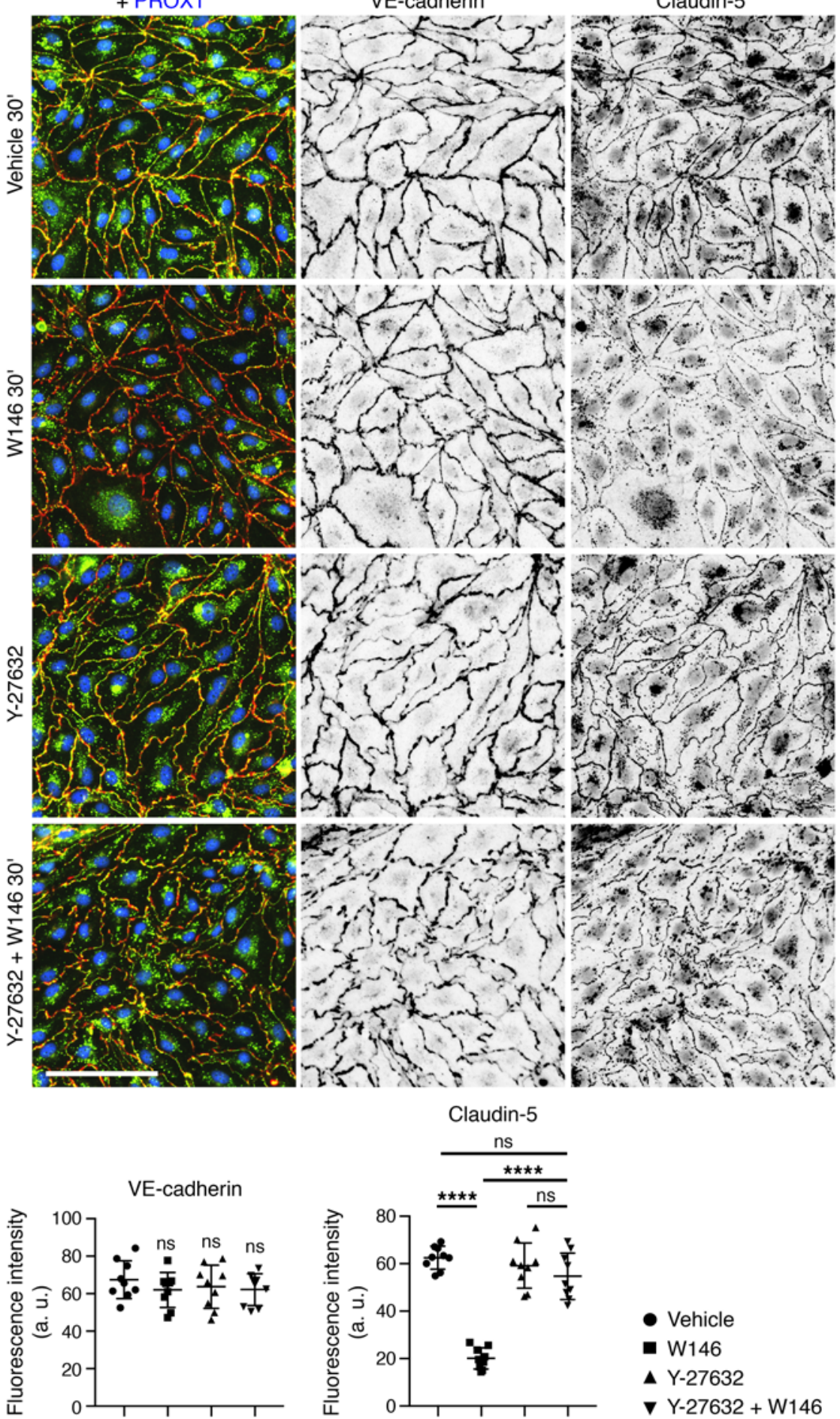

Claudin-5

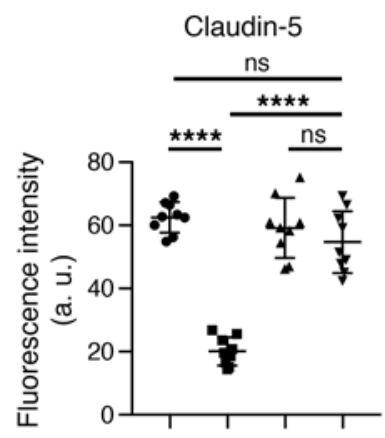

B
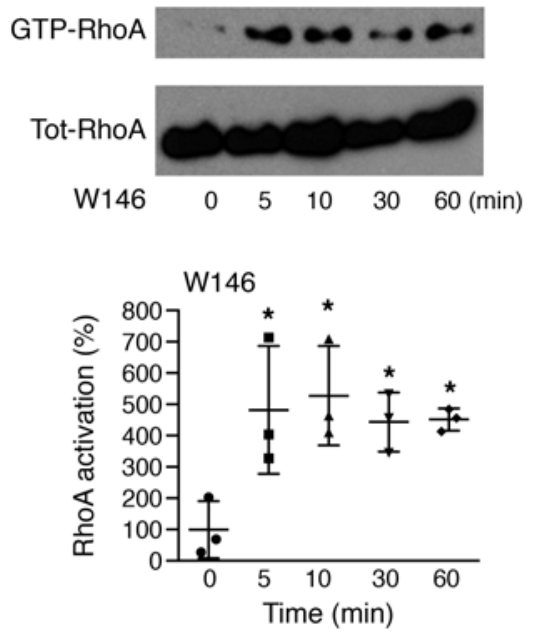

C
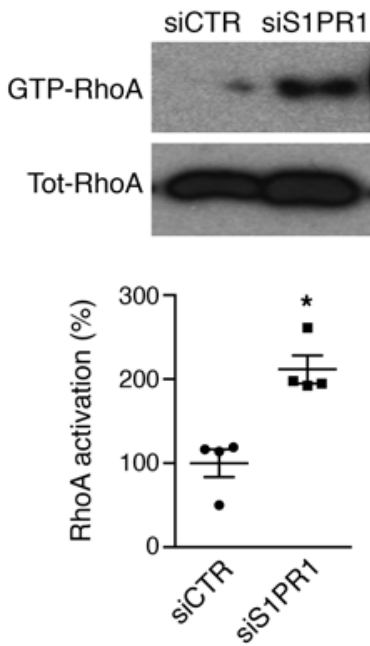

Figure 6. S1PR1 inhibits RhoA signaling to maintain claudin-5 expression in HLECs. (A) S1PR1 regulates the cell membrane localization of claudin-5 by inhibiting RhoA/ROCK signaling. VE-cadherin and claudin- 5 were localized to the cell membrane in vehicle-treated HLECs. W146 treatment for 30 minutes resulted in the dramatic downregulation of claudin-5 expression at the cell junctions. Expression of VE-cadherin was not affected, although it assumed a discontinuous (zigzag) pattern. Treatment with the ROCK inhibitor Y-27632 for 6 hours resulted in the change in the morphology of HLECs, although expression of VE-cadherin and claudin-5 were unaffected. Pretreatment with Y-27632 inhibited the W146-induced downregulation of claudin-5. (B and C) Inhibition of S1PR1 signaling in HLECs resulted in the activation of RhoA. HLECs were (B) treated with W146 for the indicated time points or (C) transfected with siS1PR1, and RhoA activity was measured using the protein lysates. RhoA activity was enhanced rapidly by both W146 and siS1PR1. $n=3$ for B and $n=4$ for C. ${ }^{*} P<0.05,{ }^{* * *} P<0.0001,1$-way ANOVA with Bonferroni's correction. Data are presented as mean \pm SEM. Scale bar: $100 \mu \mathrm{M}$. 
In conclusion, LSS regulates lymphangiogenesis via 2 distinct mechanisms of action. First, LSS inhibits the expression of prolymphangiogenic sprouting genes, such as DLL4, ANGPT2, ESM1, and ADM; this does not involve S1PR1. Second, LSS enhances VEGF-C, a process that is directly antagonized by S1PR1. Additionally, S1PR1 regulates the membrane localization of claudin-5 by inhibiting RhoA activity. Correspondingly, S1pr1 deletion in LECs results in hypersprouting and hyperbranching of lymphatic vessels, which are immature due to the delayed expression of claudin-5 and abnormal localization of VE-cadherin. Our findings are schematically summarized in Figure 7.

\section{Discussion}

We have determined that the lymphatic vasculature-specific roles of DLL4 and S1PR1 are distinct from their known activities in blood endothelial cells. While DLL4 inhibits angiogenesis $(5,37,38)$, we have determined that it promotes lymphangiogenesis. S1PR1 regulates the expression of VE-cadherin and the alignment of blood endothelial cells with respect to LSS (20-23). In contrast, S1PR1 is not required for the expression of VE-cadherin in HLECs or for the alignment of HLECs with respect to LSS. However, S1PR1 is required for the expression of claudin-5 in LECs in vitro and in vivo. S1PR1 antagonizes VEGF-A signaling in blood vascular endothelial cells (20). In contrast, S1PR1 inhibits VEGF-C signaling that is specifically enhanced by LSS. Finally, when compared with blood vascular endothelial cells, S1PR1 signaling appears to be biased toward the $\beta$-arrestin pathway in LECs.

We have also identified several previously unknown mechanisms that operate during lymphatic vascular patterning. The tip cell-expressed molecule DLL4 is an enhancer of VEGF-C signaling. Dll4 ${ }^{+/-}$embryos have hypoplastic lymphatic vessels, establishing the prolymphangiogenic role of this gene. We currently do not know whether the other tip cell-expressed molecules ADM, ANGPT2, or ESM1 could regulate VEGF-C signaling. However, evidence from published manuscripts suggests that they might. Conditional deletion of Calcrl, the gene encoding the ADM receptor, in LECs results in a lacteal phenotype similar to that of Dll4 ${ }^{+/}$ mice (51). Furthermore, $C A L C R L$ knockdown downregulates, while ADM treatment enhances, $D L L 4$ expression in cultured HLECs (51). Therefore, ADM could regulate VEGF-C signaling indirectly through DLL4. ESM1 can enhance VEGF-A signaling in blood endothelial cells (6). In addition, ESM1 is able to enhance VEGF-C-mediated HLEC proliferation (7). Finally, ectopic expression of ANGPT2 promotes lymphangiogenesis, which could be inhibited by a VEGFR3-blocking antibody (52). Thus, DLL4, ANGPT2, ADM, and ESM1 are likely part of a network that enhances VEGF-C signaling in lymphatic tip cells.

LSS is a potent inhibitor of DLL4, ADM, ESM1, and ANGPT2 expression in HLECs. Previous reports have shown that VEGF-C could transiently enhance DLL4 expression in HLECs (4). Thus, a balance between VEGF-C and LSS activities might confine the expression of DLL4 and other molecules to nonperfused tip cells (Figure 7A). The shear-responsive transcription factor KLF2 is a potential candidate through which LSS inhibits the expression of tip cell genes, as KLF2 is known to inhibit ANGPT2 and EGR1 expressions in blood vascular endothelial cells (53).

We have determined that LSS enhances VEGF-C signaling in HLECs. We have previously shown that Lyve1-Cre;Gata2 $2^{f / f l}$ and Clec2 ${ }^{-1-}$ mice, which lack lymph flow, have hypoplastic lymphatic vessels (13, 25). Nevertheless, further research is required to dissect LSS-dependent and LSS-independent pathways of VEGF-C signaling. This information could assist in the evaluation of mouse models with hypoplastic lymphatic vessels and to determine whether the LSS/VEGF-C signaling pathway is physiologically significant to promote the growth of lymphatic vessels. Mechanistically, LSS might enhance the interaction between VEGFR3 and its coreceptor NRP2 or molecules such as VEGFR2 and Integrin- $\alpha 9$. Alternatively, expression or localization of kinases or phosphatases that modulate the phosphorylation of VEGFR3 or downstream signaling molecules could be regulated by LSS.

LSS and MAPK signaling pathways are critical for the survival of quiescent blood vascular endothelial cells $(47,48,54,55)$. MAPK signaling is activated by FGF to maintain the survival and integrity of quiescent blood vascular endothelial cells (54). Similarly, deletion of Vegfc from adult mice results in the regression of lymphatic vessels (56). Therefore, we suggest that LSS/VEGF-C signaling could be important for the survival of quiescent LECs (Figure 7A). Coincidently, FGFR3 is a molecule that is upregulated by LSS in HLECs (19). Whether LSS could enhance FGF or other receptor tyrosine kinase signaling pathways remains to be determined.

In addition to promoting the growth and quiescence of LECs, LSS/VEGF-C signaling could eliminate redundant vessel segments that do not support physiologically productive lymph flow (Figure 7A). 
A

Control

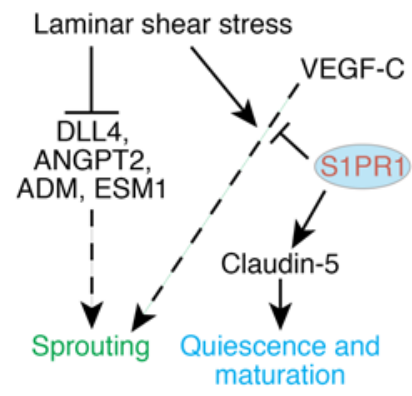

i

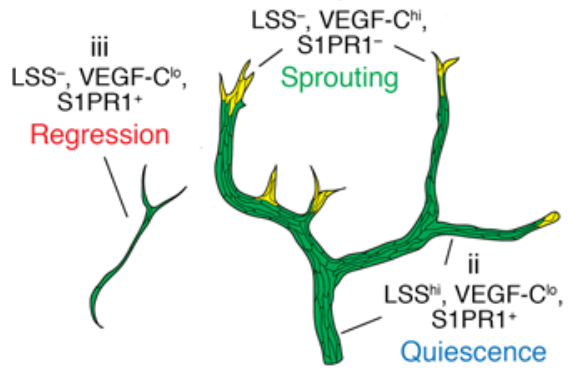

B

S1PR1 $\Delta$
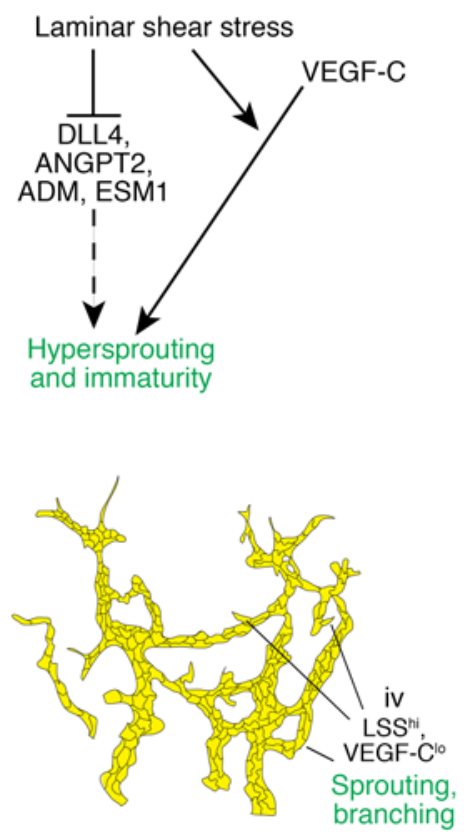

Figure 7. Model for the crosstalk between LSS, S1PR1, and VEGF-C signaling during lymphatic vascular patterning.

(A) LSS inhibits the expression of molecules such as DLL4, ANGPT2, ADM, and ESM1, thereby limiting their expression to tip cells (i). LSS enhances VEGF-C signaling to promote the survival of quiescent LECs (ii). S1PR1 functions as a buffer that prevents the hyperactivation of LSS/VECF-C signaling to prevent the sprouting of lymphatic vessels (ii). Lymphatic vessels that are exposed to a low dose of VEGF-C with little or no lymph flow regress due to reduced LSS/VEGF-C signaling (iii). (B) In the absence of S1PR1, VEGF-C signaling is hyperactive in the posterior lymphatic plexus, which results in an excessive number of sprouts and branches (iv). In addition to modulating LSS/VEGF-C signaling, S1PR1 inhibits RhoA activity to regulate the cytoskeleton and maintain claudin- 5 expression (green vessels). Consequently, mice lacking S1PR1 have immature cell junctions (yellow vessels).

Accordingly, poorly perfused lymphatic vessels will experience reduced VEGF-C/VEGFR3 signaling compared with vessels with stronger lymph flow, and they will be pruned. Successive rounds of pruning will progressively strengthen lymph flow in the remaining vessels and result in their quiescence and maturation.

S1PR1 activity is excluded from tip cells. In contrast, S1PR1 activity is enriched in more-mature lymphatic vessels, where it antagonizes LSS/VEGF-C signaling. S1PR1 activity is likely regulated by S1P that is present at high concentrations in lymph (30). We postulated that S1PR1 functions as a buffer that prevents the overactivation of the LSS/VEGF-C signaling. This model would predict excessive sprouting and insufficient pruning of lymphatic vessels in Lyve1-Cre;S1pr1 $1^{-f f}$ mice, resulting in excessive lymphatic branching, as we observed (Figure 7B).

The elongated morphology of LECs in E16.5 and older embryos suggests that they are likely exposed to LSS at these time points (Figure 2A). However, LECs could be exposed to OSS at earlier developmental time points and at branch points and valves $(16,57)$. Additionally, the excessive number of branches in the lymphatic vessels of Lyve1-Cre;S1pr1 ${ }^{-/ f l}$ mice could result in a reduction in LSS and an increase in OSS. Whether OSS could enhance VEGF-C signaling at such instances and whether S1PR1 plays a regulatory role during those processes should be investigated.

S1PR1 plays an additional role in regulating claudin-5 localization to LEC junctions in developing lymphatic vessels. Claudin-5 expression is defective in the lymphatic vessels of Lyve1-Cre;S1pr1 ${ }^{-f f l}$ mice. S1PR1-mediated inhibition of RhoA signaling appears to be important for the localization of claudin-5 to the cell membrane. Intriguingly, RhoA activity also appears to be important for LEC junctional integrity, as inhibition of ROCK results in abnormally shaped HLECs. Our results do not support a role for claudin-5 in LSS-enhanced VEGF-C signaling. However, whether the stress fibers and RhoA activity that were increased in siS1PR1-treated HLECs could be responsible for the upregulation of LSS-enhanced VEGF-C 
signaling remains to be investigated. Alternatively, the $\beta$-arrestin pathway could modulate the amplitude and kinetics of ERK signaling downstream of GPCRs (58).

VEGF-C, expression of which is enhanced by lymphedema, plays a proinflammatory role by recruiting inflammatory cells to the interstitium $(59,60)$. Inflammation, in turn, could promote interstitial fibrosis and lymphatic dysfunction $(59,61)$. Hence, approaches to promote lymphatic vessel growth without inflammation are urgently needed. S1PR1 plays a proinflammatory role by promoting $\mathrm{T}$ cell egress from lymph nodes (62). Fingolimod, which inhibits S1PR1, is used for treating the neuroinflammatory disease multiple sclerosis (63). It was recently shown that Fingolimod could inhibit inflammation and ameliorate lymphedema (64). Based on our results, we are tempted to speculate that Fingolimod likely functions by simultaneously inhibiting inflammation while promoting VEGF-C signaling. Better understanding of the relationship between S1PR1 and VEGF-C signaling could lead to the development of efficient S1PR1-targeting drugs to treat lymphedema. Furthermore, recent evidence has suggested that meningeal lymphatic vessels could regulate neuroinflammation (65). Whether Fingolimod could affect the structure and function of meningeal lymphatic vessels should be investigated.

\section{Methods}

IHC on sections and skin were performed according to our previously published protocols $(13,14,66)$. Immunocytochemistry was performed as we described recently $(13,25)$. Semiquantitative measurement of fluorescence intensities was performed using ImageJ software (NIH).

\section{Antibodies}

Primary antibodies for immunohistochemistry include rabbit anti-PROX1 (catalog 11-002, Angiobio), rat anti-mouse CD31 (catalog 553370, BD Pharmingen), rat anti-mouse VE-cadherin (catalog 550548, BD Pharmingen), chicken anti-GFP (catalog ab13970, Abcam), rabbit anti-human ZO-1 (catalog 40-2200, Invitrogen), rabbit anti-mouse CLDN5 (catalog 34-1600, Thermo Fisher Scientific), and Alexa 488-conjugated Phalloidin (catalog A12379, Invitrogen). Goat anti-human PROX1 (catalog AF2727), goat anti-mouse VEGRF3 (catalog AF743), goat anti-mouse NRP2 (catalog AF567), rat anti-mouse S1PR1 (catalog MAB7089), and goat anti-mouse LYVE-1 (catalog AF2125) were from R\&D Systems.

Secondary antibodies for IHC include Cy3-conjugated donkey anti-rabbit, Cy3-conjugated donkey anti-rat, Cy3-conjugated donkey anti-goat, Cy5-conjugated donkey anti-rat, Cy5-conjugated donkey anti-goat, FITC-conjugated donkey anti-chicken, Alexa 488-conjugated donkey anti-goat, and Biotin-conjugated donkey anti-goat antibodies, purchased from Jackson ImmunoResearch Laboratories (catalogs 711-165-152, 712-165-153, 705-165-147, 712-175-150, 705-175-147, 703-095-155, 705-545147, and 705-065-147, respectively). Alexa 488-conjugated donkey anti-rat antibody was purchased from Invitrogen (catalog A-21208).

Primary antibodies for Western blotting include mouse anti- $\beta$-Actin (catalog A5441, MilliporeSigma), rabbit anti-human GAPDH (catalog PAB13195, Abnova), rabbit anti-human S1PR1 (catalog A12935, ABclonal), and rabbit anti-human eNOS (catalog NB300-500SS, Novus Biologicals). Rabbit anti-mouse AKT (catalog 4691), rabbit anti-human phospho-AKT (catalog 4060), rabbit anti-rat ERK1/2 (catalog 4695), rabbit anti-human p38 MAPK (catalog 8690), rabbit anti-human phospho-p38 MAPK (catalog 4511), rabbit anti-human phospho-eNOS (catalog 9571), rabbit anti-human NICD (catalog 4147), rabbit anti-human DLL4 (catalog 2589), and rabbit anti-RhoA (catalog 2117) were purchased from Cell Signaling Technology.

HRP-conjugated secondary antibodies for Western blotting include goat anti-mouse IgG (catalog A 4416, MilliporeSigma) and goat anti-rabbit IgG (catalog GtxRb-003-EHRPX, ImmunoReagents).

\section{Cells}

Donwong Choi (Keck School of Medicine) provided the HLECs harvested according to his reported protocols $(15,18,19)$. HLECs were grown on culture dishes or glass slide coated with $0.2 \%$ gelatin and were maintained in EGM-2 EC Growth Medium-2 Bullet Kit (Lonza). All experiments were conducted using cells until passage 8 . HLECs were treated as potential biohazards and were handled according to institutional biosafety regulations. 


\section{Cell treatments}

S1PR1 agonist and antagonist treatment. HLECs were seeded onto 6-well plates. After 48-hour culture (80\%-100\% confluence), cells were starved for 6 hours and then treated with $20 \mu \mathrm{M}$ SEW2871 (catalog 10006440, Cayman Chemical), $10 \mu \mathrm{M}$ W146 (catalog 10009109, Cayman Chemical), or vehicle for 1 hour. Pretreated HLECs were subsequently treated with 100ng/mL VEGF-C (catalog 9199-vc-025/CF, R\&D Systems) for 10 minutes.

siRNA transfection. HLECs were seeded onto 6-well plates or culture dishes. After 24 hours of culture (around 30\% confluence), cells were transfected with siCTR (catalog 51-01-14-03, Integrated DNA Technologies), siS1PR1 (catalog SI00376201, QIAGEN), or siDLL4 (catalog hs.Ri.DLL4.13.1, Integrated DNA Technologies) using Lipofectamine RNAiMax Tranfection Reagent (catalog 13778150, Invitrogen) according to manufacturer's instruction.

VEGF-C treatment. Confluent HLECs were treated with the indicated concentrations of VEGF-C (cata$\log 9199-\mathrm{vc}-025 / \mathrm{CF}, \mathrm{R} \& \mathrm{D}$ Systems) and collected after the indicated time points.

LSS. HLECs were seeded in culture dishes and grown to $80 \%-100 \%$ confluence before exposing them to LSS for either 10 minutes or 24 hours. LSS was applied to the cells at $5 \mathrm{dynes} / \mathrm{cm}^{2}$ using the approach described previously $(18,19)$. Cells were not serum starved for VEGF-C treatment after LSS. VEGF-C was added to the dishes and incubated for 10-30 minutes for Western blotting. For quantifying EGR1 expression, VEGF-C was added to the cells and returned back to LSS for 1 hour.

ROCK inhibitor treatment. HLECs were seeded onto 24-well plates. All confluent cells were treated with $10 \mu \mathrm{M}$ Y-27632 (catalog SCM075, MilliporeSigma) in starvation media for 6 hours. The cells were then treated with either vehicle or $10 \mu \mathrm{M}$ W146 for 30 minutes. Control cells were grown in starvation media for 6 hours, followed by vehicle or $10 \mu \mathrm{M}$ W146 for 30 minutes.

\section{Cell junction analysis}

HLECs were treated with chemicals as described above, and their intracellular permeability was analyzed using In Vitro Vascular Permeability Imaging Assay (catalog 17-10398, MilliporeSigma) according to manufacturer's instructions.

\section{Lymphangiography}

Lymphangiography was performed according to a published report (52). Briefly, $2 \mu \mathrm{L}$ of $3 \%$ Evans blue (E2129, MilliporeSigma) was injected into the periorbital dermis of E17.5 embryo with a $36 \mathrm{G}$ needle attached to a Nanofil syringe (WPI, Nanofil). One minute after the injection, the embryos were imaged with a stereomicroscope.

Mice

Lyve1-Cre (30), S1prifl(31), and S1PR1-GFP (26) mice were described previously and were purchased from the Jackson Laboratory (catalogs 012601, 019141, and 026275, respectively). Mice were maintained in C57BL6 or C57BL6/NMRI mixed backgrounds. Dll4 ${ }^{+/-}$cryopreserved embryos were purchased from the Canadian Mouse Mutant Repository (CMMR), implanted into CD-1/ICR females, and then backcrossed onto a C57BL6 background $(37,67)$. Vegfr $3^{+/ E G F P}$ mice were provided in house (68).

\section{Protein isolation and analysis}

Protein was extracted from cells by using RIPA lysis buffer. Western blots were performed according to standard protocols. The protein lysate was not boiled while performing Western blot for S1PR1. The intensities of bands were measured using ImageJ software.

\section{RNA isolation and quantitative PCR}

Total RNA from HLECs was purified using Trizol (Invitrogen) according to manufacturers instructions. cDNA was synthesized from total RNA $(0.1-1.0 \mu \mathrm{g})$ with iScript Advanced cDNA Synthesis Kit (BioRad). quantitative PCR (qPCR) was performed using PowerUp SYBR Green Master Mix reagent (Applied Biosystems) in a CFX96 Real-Time System (Bio-Rad). Expression levels were normalized to GAPDH. Primer sequences are provided below.

GAPDH: 5' - GAAGGTCGGAGTCAACGGATTT - 3' and 5' - ATGGGTGGAATCATATTGGAAC - 3'; KLF2: 5' - CCTCCCAAACTGTGACTGGT - 3' and 5' - ACTCGTCAAGGAGGATCGTG - 3'; KLF4: 
5' - AGGGGGTGACTGGAAGTTGT - 3' and 5' - CCAAGCACCATCATTTAGGC - 3'; ANGPT2: 5' TCCAAGCAAAATTCCATCATTG - 3' and 5' - GCCTCCTCCAGCTTCCATGT - 3'.

The rest of the primers were ordered as predesigned primers from IDT. The catalogs of the manufacturer are as follows: EGR1, Hs.PT.58.40805543; S1PR1, Hs.PT.58.26669476; DLL4, Hs.PT.58.3416363; ADM, Hs.PT.56a.25211580; ESM1, Hs.PT.58.19279572.

\section{Rho GTPase pull-down assays}

For RhoA pull-down experiments we used the GST-RBD plasmid, a gift from Martin Schwartz (Addgene plasmid no. 15247; http://n2t.net/addgene:15247; RRID:Addgene_15247). RhoA activation in cultured cells was assessed as previously described (69). Briefly, after treatment, the cells were lysed in lysis buffer containing 20 mM HEPES (pH 7.4), 0.1M NaCl, 1\% Triton X-100, 10 mM EGTA, $40 \mathrm{mM}$ b-glycerophosphate, $20 \mathrm{mM} \mathrm{MgCl}_{2}, 1 \mathrm{mM} \mathrm{Na}_{3} \mathrm{VO}_{4}, 1 \mathrm{mM}$ dithiothreitol, $10 \mu \mathrm{g} / \mathrm{mL}$ aprotinin, $10 \mu \mathrm{g} / \mathrm{mL}$ leupeptin, and $1 \mathrm{mM}$ phenylmethylsulfonyl fluoride on ice (all from Sigma-Aldrich). The lysates were incubated with the glutathione S-transferase-rhotekin-Rho-binding domain previously bound to glutathione-Sepharose beads (Amersham Biosciences) and washed 3 times with lysis buffer. Associated GTP-bound forms of Rho were released with SDS-polyacrylamide gel electrophoresis loading buffer and analyzed by Western blot analysis using a monoclonal antibody against RhoA (catalog 2117, Cell Signaling Technology). Normalization took place based on the total RhoA levels present in the whole cell lysate of the same samples.

\section{Statistics}

For biochemical studies, $n$ refers to the number of times the experiment was performed. For histochemical analysis, $n$ refers to the total number of animals included per group. Statistically significant differences were determined using unpaired 2-tailed $t$ test or 1-way ANOVA with Bonferroni's correction. Prism software was used for statistical analyses. Data are reported as mean \pm SEM with significance set at $P<0.05$. Western blots and qPCR were performed at least 3 independent times. The most representative Western blots are presented. The average values from individual qPCR assays were presented unless otherwise stated.

\section{Study approval}

All mice were housed and handled according to the institutional IACUC protocols of Oklahoma Medical Research Foundation, Baylor college of Medicine, and Boston Children's Hospital.

\section{Author contributions}

XG, TH, and RSS conceptualized the work; XG, KY, RGA, LC, YH, KBDR, CMM, and RSS performed experiments; DC, HC, JW, HI, and TH provided critical reagents; XG and RSS wrote the manuscript with critical input from JW; and all authors provided input in designing the experiments and in editing the manuscript.

\section{Acknowledgments}

This work is supported by NIH/NHLBI (R01HL131652 to RSS; R01HL133216 to RSS and HC, R35HL135821 to TH), NIH/NIGMS COBRE (P20 GM103441 to XG; PI, Rodger McEver), Oklahoma Center for Adult Stem Cell Research (grant no. 4340) to RSS, and American Heart Association (19POST34380819 to YH). We thank Young-Kwon Hong, Wayne Orr, and Martin Schwartz for helpful suggestions and reagents, and we thank Judson Copeland for the illustration. We thank Angela Andersen, Life Science Editors, for editing the manuscript.

Address correspondence to: R. Sathish Srinivasan, 835 NE 13th Street, Oklahoma City, Oklahoma 73104, USA. Phone: 405-271-3550; Email: Sathish-Srinivasan@omrf.org.

1. Adams RH, Alitalo K. Molecular regulation of angiogenesis and lymphangiogenesis. Nat Rev Mol Cell Biol. 2007;8(6):464-478.

2. Gerhardt H, et al. VEGF guides angiogenic sprouting utilizing endothelial tip cell filopodia. J Cell Biol. 2003;161(6):1163-1177.

3. del Toro R, et al. Identification and functional analysis of endothelial tip cell-enriched genes. Blood. 2010;116(19):4025-4033. 
4. Dieterich LC, et al. DeepCAGE Transcriptomics Reveal an Important Role of the Transcription Factor MAFB in the Lymphatic Endothelium. Cell Rep. 2015;13(7):1493-1504.

5. Lobov IB, et al. Delta-like ligand 4 (D114) is induced by VEGF as a negative regulator of angiogenic sprouting. Proc Natl Acad Sci USA. 2007;104(9):3219-3224.

6. Rocha SF, et al. Esm1 modulates endothelial tip cell behavior and vascular permeability by enhancing VEGF bioavailability. Circ Res. 2014;115(6):581-590.

7. Shin JW, Huggenberger R, Detmar M. Transcriptional profiling of VEGF-A and VEGF-C target genes in lymphatic endothelium reveals endothelial-specific molecule-1 as a novel mediator of lymphangiogenesis. Blood. 2008;112(6):2318-2326.

8. Vaahtomeri K, Karaman S, Mäkinen T, Alitalo K. Lymphangiogenesis guidance by paracrine and pericellular factors. Genes Dev. 2017;31(16):1615-1634.

9. Karkkainen MJ, et al. Vascular endothelial growth factor $\mathrm{C}$ is required for sprouting of the first lymphatic vessels from embryonic veins. Nat Immunol. 2004;5(1):74-80.

10. Karkkainen MJ, et al. A model for gene therapy of human hereditary lymphedema. Proc Natl Acad Sci USA. 2001;98(22):12677-12682

11. Yao LC, et al. Pulmonary lymphangiectasia resulting from vascular endothelial growth factor-C overexpression during a critical period. Circ Res. 2014;114(5):806-822.

12. Baeyens N, Bandyopadhyay C, Coon BG, Yun S, Schwartz MA. Endothelial fluid shear stress sensing in vascular health and disease. J Clin Invest. 2016;126(3):821-828.

13. Cha B, et al. Mechanotransduction activates canonical Wnt/ $\beta$-catenin signaling to promote lymphatic vascular patterning and the development of lymphatic and lymphovenous valves. Genes Dev. 2016;30(12):1454-1469.

14. Cha B, et al. Complementary Wnt Sources Regulate Lymphatic Vascular Development via PROX1-Dependent Wnt/ $\beta$-Catenin Signaling. Cell Rep. 2018;25(3):571-584.e5.

15. Choi D, et al. Piezo1 incorporates mechanical force signals into the genetic program that governs lymphatic valve development and maintenance. JCI Insight. 2019;4(5):125068.

16. Sabine A, et al. Mechanotransduction, PROX1, and FOXC2 cooperate to control connexin37 and calcineurin during lymphatic-valve formation. Dev Cell. 2012;22(2):430-445.

17. Wang Y, et al. Syndecan 4 controls lymphatic vasculature remodeling during mouse embryonic development. Development. 2016;143(23):4441-4451.

18. Choi D, et al. ORAI1 Activates Proliferation of Lymphatic Endothelial Cells in Response to Laminar Flow Through Krüppel-Like Factors 2 and 4. Circ Res. 2017;120(9):1426-1439.

19. Choi D, et al. Laminar flow downregulates Notch activity to promote lymphatic sprouting. J Clin Invest. 2017;127(4):1225-1240

20. Gaengel K, et al. The sphingosine-1-phosphate receptor S1PR1 restricts sprouting angiogenesis by regulating the interplay between VE-cadherin and VEGFR2. Dev Cell. 2012;23(3):587-599.

21. Lee MJ, et al. Vascular endothelial cell adherens junction assembly and morphogenesis induced by sphingosine-1-phosphate Cell. 1999;99(3):301-312.

22. Yanagida K, et al. Sphingosine 1-Phosphate Receptor Signaling Establishes AP-1 Gradients to Allow for Retinal Endothelial Cell Specialization. Dev Cell. 2020;52(6):779-793.e7.

23. Jung B, et al. Flow-regulated endothelial S1P receptor-1 signaling sustains vascular development. Dev Cell. 2012;23(3):600-610.

24. Chien S. Mechanotransduction and endothelial cell homeostasis: the wisdom of the cell. Am J Physiol Heart Circ Physiol. 2007;292(3):H1209-H1224.

25. Mahamud MR, et al. GATA2 controls lymphatic endothelial cell junctional integrity and lymphovenous valve morphogenesis through miR-126. Development. 2019;146(21):dev184218.

26. Kono M, Tucker AE, Tran J, Bergner JB, Turner EM, Proia RL. Sphingosine-1-phosphate receptor 1 reporter mice reveal receptor activation sites in vivo. J Clin Invest. 2014;124(5):2076-2086.

27. Coxam B, et al. Pkd1 regulates lymphatic vascular morphogenesis during development. Cell Rep. 2014;7(3):623-633.

28. Reiter E, Ahn S, Shukla AK, Lefkowitz RJ. Molecular mechanism of $\beta$-arrestin-biased agonism at seven-transmembrane receptors. Annu Rev Pharmacol Toxicol. 2012;52:179-197.

29. Engelbrecht E, et al. Sphingosine 1-phosphate-regulated transcriptomes in heterogenous arterial and lymphatic endothelium of the aorta. Elife. 2020;9:e52690.

30. Pham TH, et al. Lymphatic endothelial cell sphingosine kinase activity is required for lymphocyte egress and lymphatic patterning. J Exp Med. 2010;207(1):17-27.

31. Allende ML, Yamashita T, Proia RL. G-protein-coupled receptor S1P1 acts within endothelial cells to regulate vascular maturation. Blood. 2003;102(10):3665-3667.

32. Dellinger MT, Meadows SM, Wynne K, Cleaver O, Brekken RA. Vascular endothelial growth factor receptor-2 promotes the development of the lymphatic vasculature. PLoS One. 2013;8(9):e74686.

33. Tzima E, et al. A mechanosensory complex that mediates the endothelial cell response to fluid shear stress. Nature. 2005;437(7057):426-431.

34. Hägerling R, et al. Distinct roles of VE-cadherin for development and maintenance of specific lymph vessel beds. $E M B O J$. 2018;37(22):e98271.

35. Yang Y, Cha B, Motawe ZY, Srinivasan RS, Scallan JP. VE-Cadherin Is Required for Lymphatic Valve Formation and Maintenance. Cell Rep. 2019;28(9):2397-2412.e4.

36. Yanagida K, et al. Size-selective opening of the blood-brain barrier by targeting endothelial sphingosine 1-phosphate receptor 1. Proc Natl Acad Sci USA. 2017;114(17):4531-4536.

37. Duarte A, et al. Dosage-sensitive requirement for mouse Dl14 in artery development. Genes Dev. 2004;18(20):2474-2478

38. Hellström M, et al. D114 signalling through Notch1 regulates formation of tip cells during angiogenesis. Nature. 2007;445(7129):776-780.

39. Gale NW, et al. Haploinsufficiency of delta-like 4 ligand results in embryonic lethality due to major defects in arterial and vascular development. Proc Natl Acad Sci USA. 2004;101(45):15949-15954.

40. Krebs LT, Shutter JR, Tanigaki K, Honjo T, Stark KL, Gridley T. Haploinsufficient lethality and formation of arteriovenous 
malformations in Notch pathway mutants. Genes Dev. 2004;18(20):2469-2473.

41. Hogan BM, et al. Vegfc/Flt4 signalling is suppressed by D114 in developing zebrafish intersegmental arteries. Development. 2009;136(23):4001-4009.

42. Bernier-Latmani J, et al. DLL4 promotes continuous adult intestinal lacteal regeneration and dietary fat transport. J Clin Invest. 2015;125(12):4572-4586.

43. Zheng W, et al. Notch restricts lymphatic vessel sprouting induced by vascular endothelial growth factor. Blood. 2011;118(4):1154-1162.

44. Niessen K, et al. The Notch1-D114 signaling pathway regulates mouse postnatal lymphatic development. Blood. 2011;118(7):1989-1997.

45. Dellinger M, et al. Defective remodeling and maturation of the lymphatic vasculature in Angiopoietin-2 deficient mice. Dev Biol. 2008;319(2):309-320

46. Fritz-Six KL, Dunworth WP, Li M, Caron KM. Adrenomedullin signaling is necessary for murine lymphatic vascular development. J Clin Invest. 2008;118(1):40-50.

47. Akimoto S, Mitsumata M, Sasaguri T, Yoshida Y. Laminar shear stress inhibits vascular endothelial cell proliferation by inducing cyclin-dependent kinase inhibitor p21(Sdi1/Cip1/Waf1). Circ Res. 2000;86(2):185-190.

48. Lin K, et al. Molecular mechanism of endothelial growth arrest by laminar shear stress. Proc Natl Acad Sci USA. 2000;97(17):9385-9389.

49. Baeyens N, et al. Defective fluid shear stress mechanotransduction mediates hereditary hemorrhagic telangiectasia. J Cell Biol. 2016;214(7):807-816

50. Kjoller L, Hall A. Signaling to Rho GTPases. Exp Cell Res. 1999;253(1):166-179.

51. Davis RB, Kechele DO, Blakeney ES, Pawlak JB, Caron KM. Lymphatic deletion of calcitonin receptor-like receptor exacerbates intestinal inflammation. JCI Insight. 2017;2(6):e92465.

52. Zheng W, et al. Angiopoietin 2 regulates the transformation and integrity of lymphatic endothelial cell junctions. Genes Dev. 2014;28(14):1592-1603.

53. Parmar KM, et al. Integration of flow-dependent endothelial phenotypes by Kruppel-like factor 2. J Clin Invest. 2006;116(1):49-58. 54. Murakami M, et al. The FGF system has a key role in regulating vascular integrity. J Clin Invest. 2008;118(10):3355-3366.

55. Ricard N, et al. Endothelial ERK1/2 signaling maintains integrity of the quiescent endothelium. J Exp Med. 2019;216(8):1874-1890.

56. Nurmi H, Saharinen P, Zarkada G, Zheng W, Robciuc MR, Alitalo K. VEGF-C is required for intestinal lymphatic vessel maintenance and lipid absorption. EMBO Mol Med. 2015;7(11):1418-1425.

57. Sweet DT, et al. Lymph flow regulates collecting lymphatic vessel maturation in vivo. J Clin Invest. 2015;125(8):2995-3007.

58. Gutkind JS, Kostenis E. Arrestins as rheostats of GPCR signalling. Nat Rev Mol Cell Biol. 2018;19(10):615-616.

59. Ho YC, Srinivasan RS. Lymphatic Vasculature in Energy Homeostasis and Obesity. Front Physiol. 2020;11:3.

60. Karaman S, et al. Transgenic overexpression of VEGF-C induces weight gain and insulin resistance in mice. Sci Rep. 2016;6:31566

61. Kataru RP, et al. Fibrosis and secondary lymphedema: chicken or egg? Transl Res. 2019;209:68-76.

62. Pham TH, Okada T, Matloubian M, Lo CG, Cyster JG. S1P1 receptor signaling overrides retention mediated by G alpha i-coupled receptors to promote $\mathrm{T}$ cell egress. Immunity. 2008;28(1):122-133.

63. Brinkmann V, et al. The immune modulator FTY720 targets sphingosine 1-phosphate receptors. J Biol Chem. 2002;277(24):21453-21457.

64. García Nores GD, et al. CD4 ${ }^{+} \mathrm{T}$ cells are activated in regional lymph nodes and migrate to skin to initiate lymphedema. Nat Commun. 2018;9(1):1970.

65. Louveau A, et al. CNS lymphatic drainage and neuroinflammation are regulated by meningeal lymphatic vasculature. Nat Neurosci. 2018;21(10):1380-1391.

66. Geng X, et al. Multiple mouse models of primary lymphedema exhibit distinct defects in lymphovenous valve development. Dev Biol. 2016;409(1):218-233.

67. Herman AM, Rhyner AM, Devine WP, Marrelli SP, Bruneau BG, Wythe JD. A novel reporter allele for monitoring Dll4 expression within the embryonic and adult mouse. Biol Open. 2018;7(3):bio026799.

68. Ichise T, Yoshida N, Ichise H. H-, N- and Kras cooperatively regulate lymphatic vessel growth by modulating VEGFR3 expression in lymphatic endothelial cells in mice. Development. 2010;137(6):1003-1013.

69. Zahra FT, et al. Endothelial RhoA GTPase is essential for in vitro endothelial functions but dispensable for physiological in vivo angiogenesis. Sci Rep. 2019;9(1):11666 